\title{
16. RADIOLARIANS FROM THE NORTH ATLANTIC OCEAN, DEEP SEA DRILLING PROJECT LEG 941
}

\author{
M. J. Westberg-Smith, L. E. Tway, and W. R. Riedel, Geological Research Division, \\ Scripps Institution of Oceanography²
}

\begin{abstract}
Radiolarians occur at all Leg 94 sites in Quaternary and Pliocene sediments, and at Sites 608 and 610 in Miocene sediments. At Sites 607 and 609 there is an inverse relation between abundance of terrigenous components (in the siltsand range) and both the abundance and preservation of radiolarians, although this tendency has more exceptions at Site 607. Cluster analysis indicated a substantial change in the radiolarian assemblage at the level of Core 609-12 or -13, but no enduring change was detected in the samples of equivalent age at Site 607 .
\end{abstract}

\section{INTRODUCTION}

DSDP Leg 94 drilled six sites in the mid-Atlantic between $37^{\circ}$ and $53^{\circ} \mathrm{N}$ :

\begin{tabular}{ccc}
\hline Site & Location & $\begin{array}{c}\text { Water depth } \\
(\mathrm{m})\end{array}$ \\
\hline 606 & $37^{\circ} 20.29^{\prime} \mathrm{N}, 35^{\circ} 30.02^{\prime} \mathrm{W}$ & 3007 \\
607 & $41^{\circ} 00.07^{\prime} \mathrm{N}, 32^{\circ} 57.44^{\prime} \mathrm{W}$ & 3426 \\
608 & $42^{\circ} 50.21^{\prime} \mathrm{N}, 23^{\circ} 05.25^{\prime} \mathrm{W}$ & 3526 \\
609 & $49^{\circ} 52.67^{\prime} \mathrm{N}, 24^{\circ} 14.29^{\prime} \mathrm{W}$ & 3883 \\
610 & $53^{\circ} 13.47^{\prime} \mathrm{N}, 18^{\circ} 53.69^{\prime} \mathrm{W}$ & 2445 \\
611 & $52^{\circ} 50.47^{\prime} \mathrm{N}, 30^{\circ} 18.58^{\prime} \mathrm{W}$ & 3203 \\
\hline
\end{tabular}

Radiolarians are present in the Pleistocene and Pliocene sediments at these sites and in some of the Miocene sediments of Sites 608 and 610. Figure 1 summarizes the occurrences of calcareous and siliceous fossils in Leg 94 cores.

\section{PROCEDURES}

Samples were acidified, sieved at $44 \mu \mathrm{m}$, and the residue pipetted onto slides and mounted in Canada balsam. Relative abundances of counting groups shown in Table 1 are recorded as percentages of approximately 600 radiolarians counted in diagonal traverses across the slide. Abundance estimates of Miocene species are presented in Tables 2 and 3.

\section{RESULTS}

\section{Site 606}

Site 606 consists of two piston-core holes located on the upper western flank of the Mid-Atlantic Ridge. Corecatcher samples from Hole 606 were examined for radiolarians.

Radiolarians are present in the Quaternary and Pliocene sediments of the first ten cores of Hole 606. Most

\footnotetext{
${ }^{1}$ Ruddiman, W. F., Kidd, R. B., Thomas, E., et al., Init. Repts. DSDP, 94: Washington (U.S. Govt. Printing Office).

2 Address: Geological Research Division, A-020, Scripps Institution of Oceanography, University of California, La Jolla, CA 92093 (reprint requests should be addressed to M. J. Westberg-Smith $\mathrm{c} / \mathrm{o}$ W. R. Riedel at this address).
}

of the species in these samples are long-ranged forms that provide little stratigraphic information. However, there are several species present whose ranges are confined to the upper Pliocene and Pleistocene: Amphirhopalum ypsilon, Didymocyrtis tetrathalamus, and Theocorythium trachelium. The latest occurrence of Stylatractus universus occurs between Samples 606-1,CC and 606-2,CC.

Preservation is very good in the first two core catchers and the assemblage is diverse, containing forms that inhabit warm-temperate surface waters, such as: collosphaerids, phacodiscids, artiscins, pyloniids, Pterocorys zancleus, Lithopera bacca, and Phormostichoartus corbula. In the sample from Core 606-3, the radiolarians are less well preserved and less diverse, and in the next core, 606-4, siliceous fossils are dissolved. In the core catchers from Cores 606-5 and -6 , radiolarians are moderately well preserved, but they are rare and not very diverse. In core catchers from Cores 606-7 through -10, radiolarians are more abundant, diverse, well preserved, and include the warm-temperate, surface forms found in the top of this hole. All samples from below Core 606-10 were barren of radiolarians.

\section{Site 607}

Two holes were piston cored at Site 607, in the uppermiddle western flank of the Mid-Atlantic Ridge. Radiolarians are present and well preserved in most of the upper Pliocene to Pleistocene samples examined from Holes 607 and 607A, and in several upper Miocene samples from Hole 607A. The latest occurrence of Stylatractus universus occurs between Samples 607-2-6, 40-42 cm and $607-1, \mathrm{CC}$; and between Samples 607A-3,CC and 607A$2, \mathrm{CC}$. Samples that contain abundant, diverse assemblages of radiolarians, including species considered indicative of warm-temperate surface water (collosphaerids, artiscins, pyloniids, Pterocanium charybdeum, Phormostichoartus corbula, and others), are interspersed with samples that contain only rare, long-ranging species believed to inhabit cold or deep water (robust actinommids, spongodiscids, Cornutella sp., Cyrtopera laguncula), or in which radiolarians are entirely absent. Abundance and 


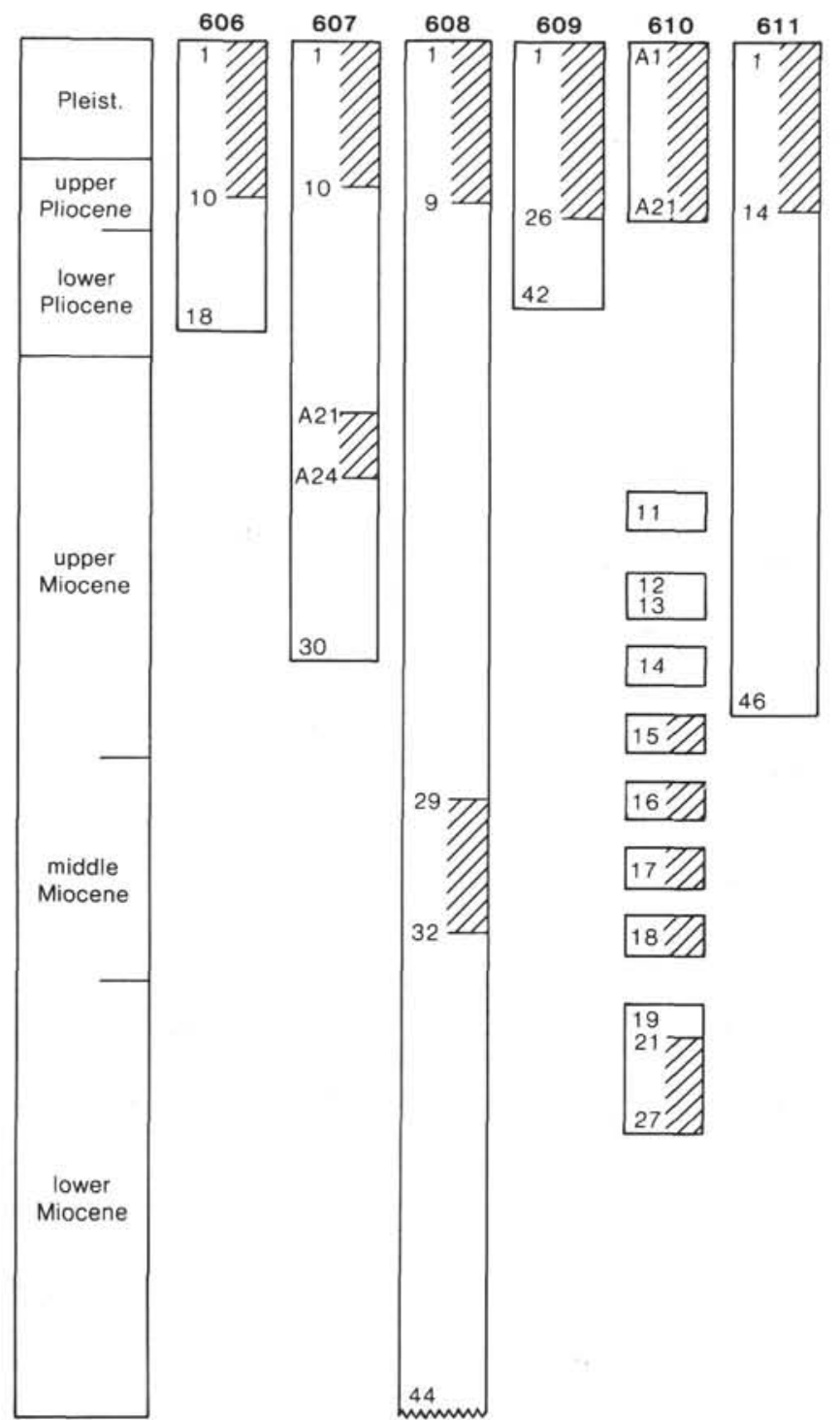

Figure 1. Neogene sediments cored on Leg 94. Core sites are represented by numbered rectangles. Empty areas indicate sediments containing only calcareous microfossils, hachured areas indicate the presence of both calcareous and siliceous microfossils. Note that Hole 608 continues down through the Paleogene, but no siliceous microfossils were found below the Neogene.

preservation of radiolarians are shown in Figure 2, and discussed later in this chapter. Table 1 shows percent abundances of counting groups from some of these samples.

In the core catchers from the Pliocene and Pleistocene Hole $607 \mathrm{~A}$ samples, radiolarians are very rare or absent below Core 607A-9, except for Sample 607A-12,CC. Radiolarians are present in upper Miocene Samples 607A$23, \mathrm{CC}$ and $-24, \mathrm{CC}$, but only in $-24, \mathrm{CC}$ are they abundant and diverse. Stichocorys peregrina is common in this sample, therefore it belongs in either the $S$. peregrina Zone or the Spongaster pentas Zone.

\section{Site 608}

Two holes were piston cored at Site 608 on the southern flank of the King's Trough tectonic complex. Because the cores from the two holes could be expected to be closely similar, radiolarians were not investigated in both. Samples from Miocene, upper Pliocene, and Pleistocene sediments of Hole 608 contain radiolarian assemblages. In the Pliocene and Pleistocene cores, intervals with well-preserved, diverse assemblages alternate with intervals in which there are only robust, deep-living radiolarians or in which there are no siliceous fossils. The only radiolarian event observed in these cores is the latest occurrence of Stylatractus universus which occurs between Samples 608-2,CC and 608-1,CC.

With the exception of a few forms in Sample 608$11, \mathrm{CC}$, radiolarians are absent from Section 608-9-3 down through Core 608-28. All eight samples examined between Samples 608-29-2, 40-42 cm and 608-32-5, 40-42 $\mathrm{cm}$ contain species representative of the middle Miocene Dorcadospyris alata Zone. Table 2 shows the order of magnitude abundances of these species. Below Core $608-33$, all the samples that were examined are barren of siliceous fossils.

\section{Site 609}

Site 609 is located on the upper-middle eastern flank of the Mid-Atlantic Ridge, and consists of four holes, one of which is reported on here.

Radiolarians are present in the upper Pliocene to Pleistocene sediments of Site 609. Preservation of radiolarians throughout these cores is generally good, but in many samples, dilution by glacial debris is so great that very few or no radiolarians are observed on the strewn slides. In Figure 2, which presents abundance of radiolarians from Hole 609, every sample shown to contain few or no radiolarians has abundant glacial debris in the strewn slides of the fraction $>44 \mu \mathrm{m}$. Samples with abundant, well-preserved assemblages can be found down through Sample 609-26,CC. Orosphaerid fragments and occasional robust forms were found in a few samples between Cores 27 and 30, and sediments below that level are barren of siliceous fossils.

Most of the forms found in Hole 609 are long-ranging species, but Amphirhopalum ypsilon, Theocorythium trachelium, and $T$. vetulum, which are characteristic of Pliocene to Pleistocene assemblages, are present in many of the samples above Core 609-15. Percent abundances of radiolarian counting groups in Cores 609-1 through 17 are recorded in Table 1.

\section{Site 610}

Site 610 is located on the western side of the Rockall Trough at the crest of the sediment drift, Feni Ridge. Six holes were drilled at this site, two of which were examined for this report.

Radiolarians are present in the Pleistocene, upper Pliocene, and Miocene sediments of Site 610. The glacial cycles of the Pliocene and younger cores yield samples that alternate between very well-preserved, abundant, diverse assemblages, and sparse assemblages of a few robust taxa and samples in which there are no radiolarians at all. In Hole 610A, radiolarians were found in at least one sample from nearly every core down to 610A-21,CC.

The Miocene cores also contain radiolarian assemblages in varying degrees of abundance and preserva- 

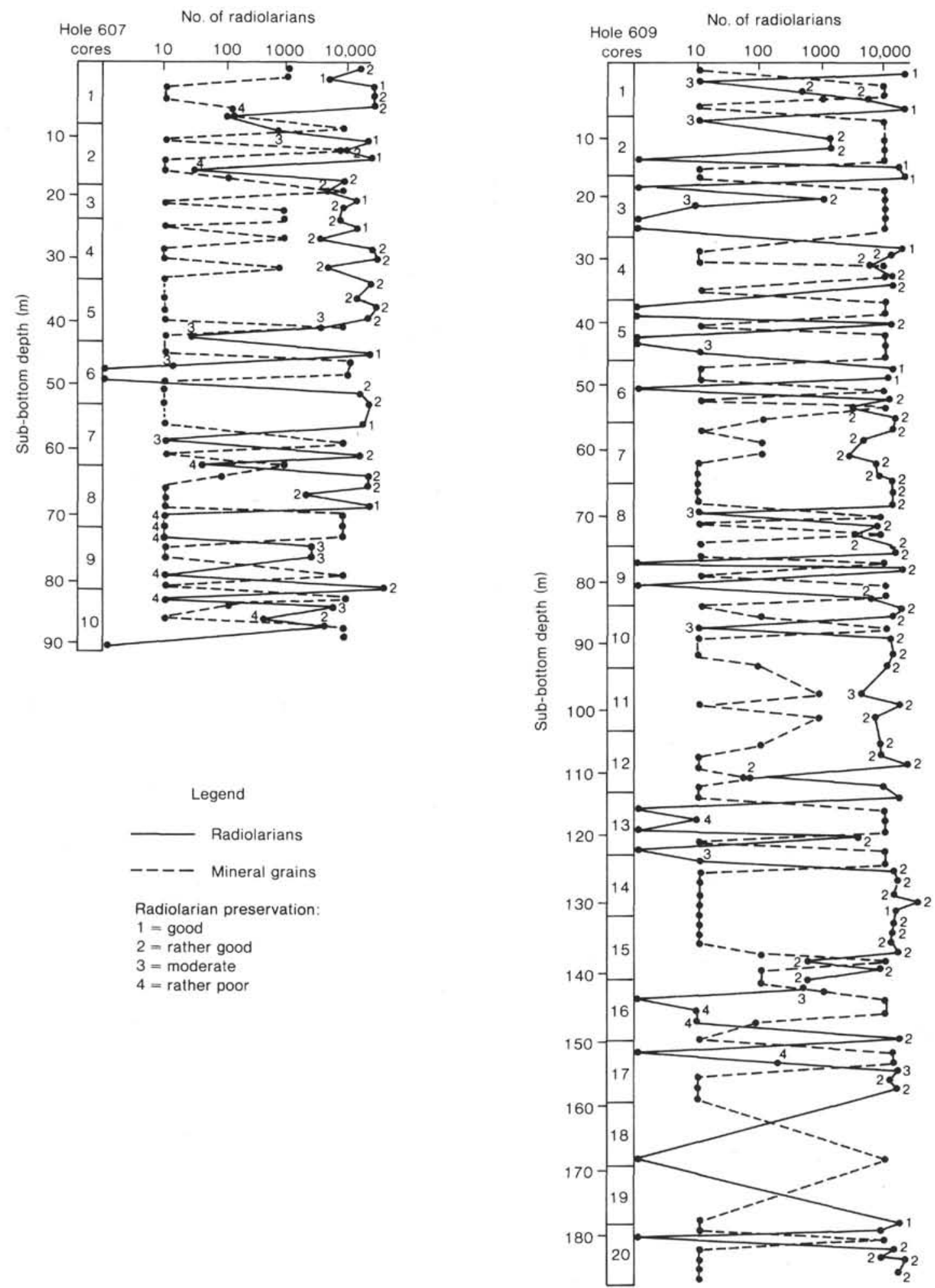

Figure 2. Numbers of radiolarians and mineral grains, and radiolarian preservation on strewn slides of the $>44-\mu \mathrm{m}$ fraction at Sites 607 and 609.

tion. Sample $610-15-2,46-48 \mathrm{~cm}$ is placed in the Didymocyrtis antepenultima Zone based on the presence of that species and absence of its ancestor, D. laticonus. Samples from Cores $610-16,-17$, and -18 contain species characteristic of the middle Miocene Diartus petterssoni and Dorcadospyris alata Zones. Although the following species are found in small numbers, each occurs in at least two consecutive samples in this interval: Didymocyrtis laticonus, Dorcadospyris alata, Lithopera renzae, and $L$. neotera. In Cores 610-19 and -20, radiolarians are either too rare and poorly preserved for age determination or they are entirely dissolved. Between Samples 
Table 1. Abundance of radiolarian counting groups at Sites 607 and 609.

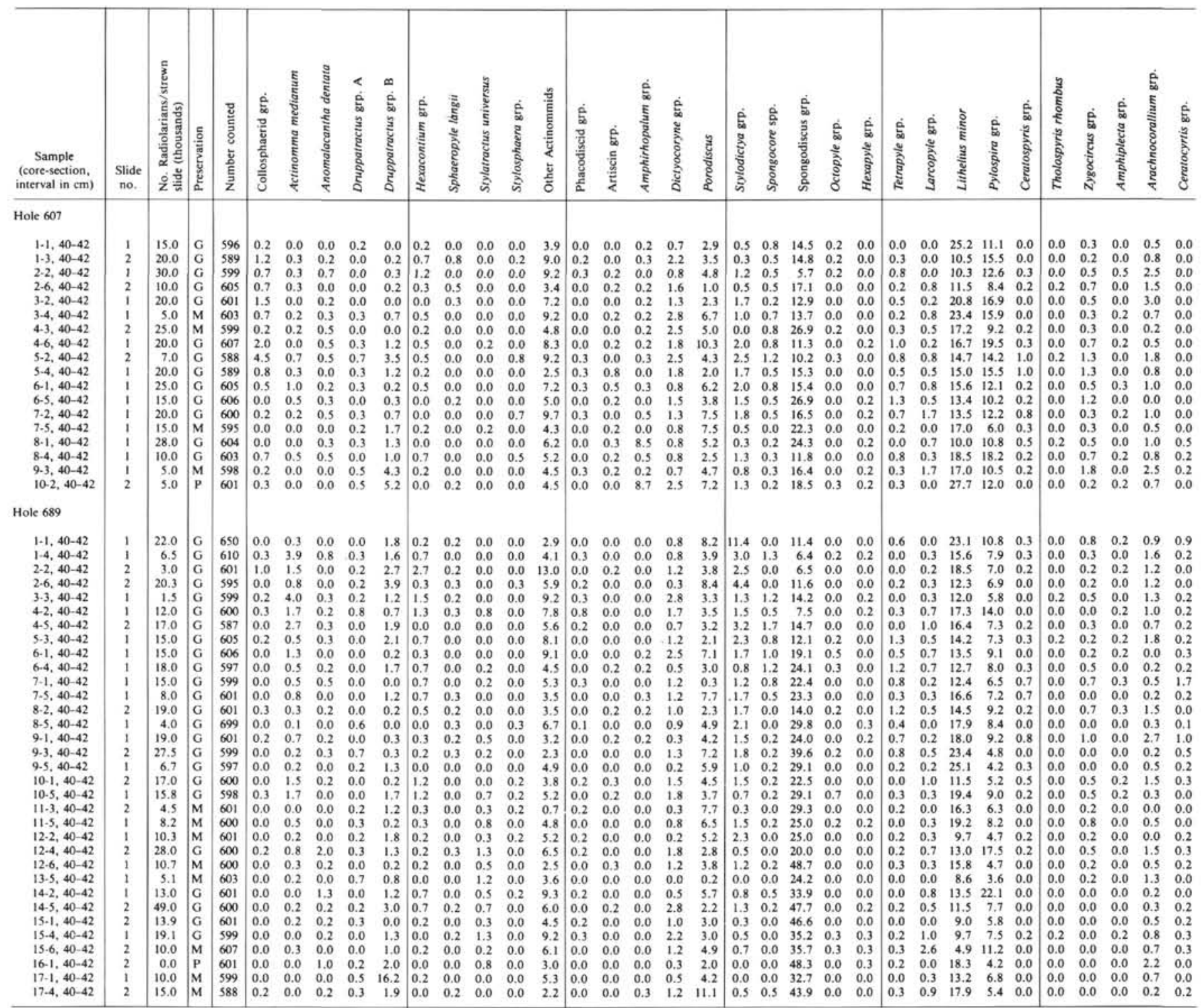

Note: The third column shows the estimated number of radiolarians on the strewn slide. Preservation (fourth column): $\mathrm{G}=$ good, $\mathrm{M}=$ moderate, and $\mathrm{P}=$ poor. The fifth column shows the actual number counted to derive the percentages that make up the remainder of the table.

610-21-2, 46-48 cm and 610-27,CC, radiolarian assemblages are more abundant and moderately well-preserved, and appear to be from the Cyrtocapsella tetrapera Zone of the early Miocene. This age is based on the presence of C. tetrapera, C. cornuta, and Lychnocanoma elonga$t a$, and the absence of Stichocorys delmontensis and $S$. wolffii. In Samples 610-21-2, 46-48 cm and 610-26-3, $70-72 \mathrm{~cm}$ there are single, reworked specimens of the late Eocene Lithocyclia aristotelis group. Abundances of Miocene species are recorded in Table 3.

\section{Site 611}

Site 611 consists of six holes drilled on the northeastern edge of Gardar Drift along the eastern flank of the Reykjanes Ridge.

Radiolarians are well-preserved, but generally not very abundant in some of the samples examined from Hole
611. Core catchers of Cores 611-2, -5, -6, and -7 are barren or contain only occasional specimens. Very sparse assemblages were found in Cores $611-8,-12$, and -14 , and Cores $611-1,-3,-4,-11$, and -13 have more abundant and diverse assemblages. In the Pliocene to upper Miocene sediments below Core $611 \mathrm{C}-15$, radiolarians are very rare or absent, with the exception of Cores $611 \mathrm{C}$ $18,-20$ and -27 . Site 611 is reported on in another chapter in this volume (Morley, this volume).

\section{ABUNDANCE AND PRESERVATION OF RADIOLARIANS}

Abundances of radiolarians on slides prepared routinely from unweighed samples were estimated according to the procedure described by Riedel and Sanfilippo (1978, pp. 82-83), and preservation was recorded according to the numbered scale established by Westberg and 
Table 1 (continued).

\begin{tabular}{|c|c|c|c|c|c|c|c|c|c|c|c|c|c|c|c|c|c|c|c|c|c|c|c|c|c|c|c|c|c|c|c|c|c|c|c|}
\hline & 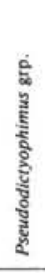 & 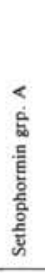 & 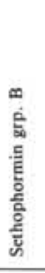 & 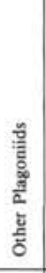 & 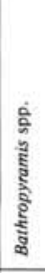 & 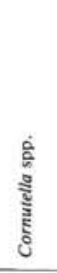 & 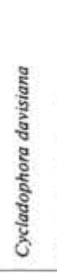 & 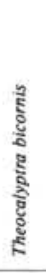 & 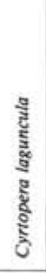 & 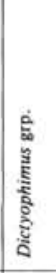 & $\begin{array}{l}\text { Eे } \\
\text { s. } \\
\text { है } \\
\text { है है } \\
\text { है }\end{array}$ & 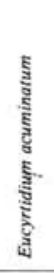 & $\begin{array}{l}\text { है } \\
\text { है } \\
\text { है } \\
\text { प्र }\end{array}$ & 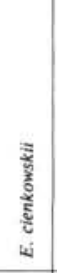 & 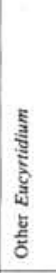 & 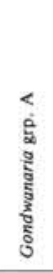 & 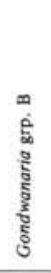 & 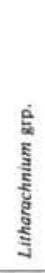 & 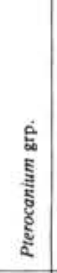 & 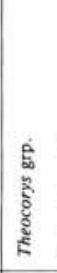 & 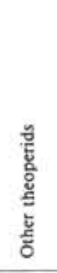 & 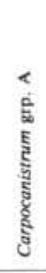 & 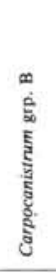 & 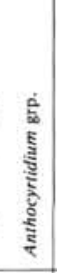 & 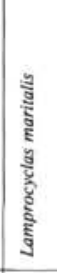 & 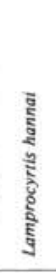 & 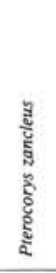 & 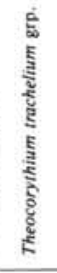 & 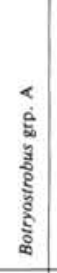 & $\begin{array}{l}\text { हैँ } \\
\text { हैँ } \\
\text { हूँ } \\
\infty \\
\infty\end{array}$ & 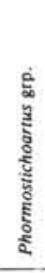 & 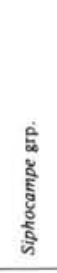 & 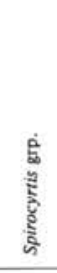 & 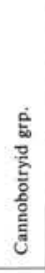 & 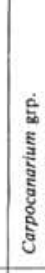 \\
\hline & & & & & & & & & & 0 & & & & & & & & & & & & & & & & & & & & & & & & & \\
\hline 3. & 3.8 & . & 0.8 & & 0 & 2 & 15.1 & 0. & 2 & 0.2 & 0.3 & & .0 & 0.0 & & & & & & & 0.3 & 0.7 & & & 2 & & & & 1.8 & 0 & & 3 & & 5 & 2 \\
\hline$\therefore$ & 7.8 & 0.2 & 0.3 & 0.0 & 0.2 & .8 & 7.5 & 0.0 & 2 & 0.2 & 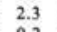 & & 0.0 & 0.0 & & 0.0 & 0.0 & 0 & 3 & 2 & 1.3 & 2.3 & 0 & .2 & 0.0 & 0.0 & 0.8 & 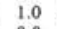 & 4.5 & 0.0 & 5 & 3 & 0 & .2 & .2 \\
\hline 5,7 & 6.6 & 0.0 & 0.0 & & 0.0 & & 27.3 & 0. & 3 & 0. & & & 0.0 & 0.0 & & & 0.0 & 8 & 0 & 3 & 0.8 & $?$ & & 0.0 & 0 & 0 & 2 & .0 & 1.5 & 0 & & 0 & 0 & 7 & s. \\
\hline 3.4 & 5.7 & 0.0 & 0.0 & & 0.0 & 1.0 & 5.2 & & 5 & 0. & 0. & & 0.2 & n & & & 0.0 & 0 & & & 1. & 0 & & 0 & 0.3 & 0.0 & & & & .0 & & 0 & 7 & $\begin{array}{l}0 \\
0\end{array}$ & 5 \\
\hline $\begin{array}{l}3.5 \\
20\end{array}$ & 1.8 & $\begin{array}{l}0.0 \\
0.0\end{array}$ & $\begin{array}{l}0.0 \\
0.0\end{array}$ & $\begin{array}{l}0.0 \\
0.2\end{array}$ & $\begin{array}{l}0.0 \\
0.2\end{array}$ & 7.7 & $\begin{array}{r}5.5 \\
21.5\end{array}$ & & $\begin{array}{l}0 \\
0\end{array}$ & $\begin{array}{l}0.4 \\
0 .\end{array}$ & 0.0 & & 0.0 & 0.0 & & & 0.0 & 0.3 & & & 0.3 & ? & & .0 & & & & & & 0 & & 3 & .0 & 0 & 8 \\
\hline 0.0 & $\begin{array}{l}1.0 \\
3.7\end{array}$ & $\begin{array}{l}0.0 \\
1.7\end{array}$ & $\begin{array}{l}0.0 \\
0.0\end{array}$ & $\begin{array}{l}0.2 \\
0.0\end{array}$ & $\begin{array}{l}0.2 \\
0.0\end{array}$ & 0.2 & $\begin{array}{r}21.1 \\
1.0\end{array}$ & $\begin{array}{l}0.0 \\
3.8\end{array}$ & $\begin{array}{l}0 \\
0 \\
0\end{array}$ & $\begin{array}{l}0.2 \\
0.8\end{array}$ & 0. & & $\begin{array}{l}0.2 \\
0.2\end{array}$ & 0.0 & & & 0.0 & 0.2 & $\begin{array}{l}.7 \\
7\end{array}$ & & 0. & $\begin{array}{l}7 \\
7\end{array}$ & & $\begin{array}{l}0.0 \\
0.8\end{array}$ & & 0 & & & $\begin{array}{l}2.0 \\
3.2\end{array}$ & $\begin{array}{l}0.0 \\
0.0\end{array}$ & & ${ }_{0}^{0}$ & 0 & & 2.2 \\
\hline 3.8 & 3.5 & 0.0 & 0.2 & 0.3 & 0.2 & $\begin{array}{l}0.3 \\
1.7\end{array}$ & $\begin{array}{l}1.0 \\
2.0\end{array}$ & $\begin{array}{l}3.8 \\
0.0\end{array}$ & 0 & $\begin{array}{l}0.0 \\
0.3\end{array}$ & $\begin{array}{l}0.5 \\
0.2\end{array}$ & 0 & $\begin{array}{l}0.2 \\
0.0\end{array}$ & 0.0 & & & $\begin{array}{l}0.0 \\
0.0\end{array}$ & & $\begin{array}{l}7 \\
5\end{array}$ & & & & & $\begin{array}{l}0.8 \\
0.0\end{array}$ & & & & & $\begin{array}{l}2 \\
3 \\
3\end{array}$ & .2 & & & $\begin{array}{l}0.0 \\
0.0\end{array}$ & s. & 1.0 \\
\hline & 4.7 & 0.0 & 0.2 & 0.0 & 0.2 & 0.8 & 17.8 & 0.8 & & O & 0. & & 0 & 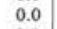 & & & 0.0 & & & & & & & & & 2 & & & 3.2 & OR & & 2 & , & 2 & .3 \\
\hline & 4.4 & 0. & 0.7 & 0.5 & 0.2 & 13 & 8.0 & & 2 & 0 & & & 0.0 & & & & 0.0 & & 3 & & 3 & 5 & & 0.5 & & 0 & & & 22 & 0.2 & & 0 & & & 8 \\
\hline 1.8 & 3.8 & 0. & 0.3 & 0.0 & 0.2 & 0.3 & 16.7 & 0. & 0 & 0.2 & 0 & & 0.0 & 0 & & 2 & 0.0 & 3 & 0.2 & & 0.0 & 2 & & 0.2 & & 0 & & & 4.0 & 0 & & & 0.0 & .0 & 5 \\
\hline & 3.2 & 0. & 0.0 & & & 3 & 2.0 & & 0 & 0. & & & 0.2 & 0. & & & 0.2 & & & & & & & & & & & & & 0 & & & & 0 & .2 \\
\hline & 3.3 & 0. & 0.0 & & & & 17.5 & & & & & & & & & & & & & & & & & & & & & & & & & & & & .5 \\
\hline & 4.2 & 0. & $\begin{array}{l}0.0 \\
0.2\end{array}$ & & $0_{0}^{0}$ & $\begin{array}{l}0.5 \\
25\end{array}$ & $\begin{array}{r}16.8 \\
60\end{array}$ & & & & & & & & & & & & & & & & & & & & & & & & & & & & 3 \\
\hline 7. & $\begin{array}{l}5.2 \\
6.3\end{array}$ & $\begin{array}{l}0.4 \\
0.8\end{array}$ & $\begin{array}{l}0.2 \\
0.3\end{array}$ & $\begin{array}{l}0.0 \\
0.0\end{array}$ & $\begin{array}{l}0.2 \\
0.3\end{array}$ & $\begin{array}{l}2.5 \\
0.3\end{array}$ & $\begin{array}{l}6.0 \\
4.2\end{array}$ & . & & & & & & & & & & & & & & & & 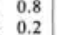 & & & & & & ${ }_{0}^{0}$ & & & 0 & & 3 \\
\hline 2.8 & 2.2 & 0.2 & 0.2 & 0.0 & 0.0 & 0.8 & & 0.2 & 0.0 & 0.0 & 0.0 & 0.0 & 0.2 & 0.0 & & 0.0 & 0.0 & 0.2 & 3 & & 0.0 & 2.0 & 0.3 & $\begin{array}{l}0.2 \\
1.0\end{array}$ & & & 0.0 & 0.4 & 2.3 & .0 & 0.0 & 0.2 & 0.0 & .0 & 0.0 \\
\hline & & & & & & & & & & & & & & & & & & & & & & & & & & & & & & & & & & & \\
\hline & & & & & & & & & & & & & & & & & & & & & & & & & & & & & & & & & & & \\
\hline & 8.3 & 0. & 0.7 & 0.5 & 0 & & 4.2 & & & & & & & & & & & & & & & & & & & & & & & 1 & & & & & \\
\hline & 3.4 & . & 0.0 & 0.2 & & 1.2 & 17.3 & & & & & & & & & & & & & & & & & & & & & & & 2 & & & & & \\
\hline & & 0. & 0.0 & & 0 & 1.2 & & & & & & & & 0 & & & & & & & & & & & & & & & & & & & & & \\
\hline & 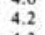 & 0. & 0.5 & & & & & & & 0 & & & & 0 & & & & & & & & & & & & & & & & & & & & & \\
\hline & 4. & 0 & 0. & & & & & & & 0 . & & & & & & & & & & & & & & & & & & & & & & & & & \\
\hline & 3. & 0 & 0 . & & & & & & & 0 . & & & & & & & & & & & & & & & & & & & & & & & & & \\
\hline ?. & 2. & ( & 0 . & & & & 10 & & & 0 & 0 & & & & & & & & & & & & & & & & & & & & & & & & \\
\hline & 8 & 0. & 0. & & & & & & & & & & & & & & & & & & & & & & & & & & & & & & & & \\
\hline & 5.8 & 0. & 0. & 0 & & & & & & & 1 & & & & & & & & & & & & & & & & & & & & & & & & \\
\hline & & $\begin{array}{l}0 . \\
0\end{array}$ & $\begin{array}{l}0.4 \\
0.8\end{array}$ & 0 & & & & & & & & & & & & & & & & & & & & & & & & & & & & & & & \\
\hline & 0 & . & $\begin{array}{l}0.0 \\
0.0\end{array}$ & $\begin{array}{l}0.3 \\
0.0\end{array}$ & 0 & 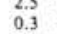 & $\begin{array}{l}10.5 \\
15.2\end{array}$ & 0 & & 0. & 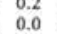 & & & & & & & & & & & & & & & & & & & & & & & & \\
\hline & & & & & & & & & & & & & & & & & & & & & & & & & & & & & & & & & & & \\
\hline & & & & & & & & & & & & & & & & & & & & & & & & & & & & & & & & & & & \\
\hline & & & & 0. & & & 12.7 & & & & & & & & & & & & & & & & & & & & & & & & & & & & \\
\hline & & & 0 & 0. & & & & & & & & & & & & & & & & & & & & & & & & & & & & & & & \\
\hline & & & 0 & ${ }_{0 .}^{0}$ & 0. & & 29 & 0 & & & & & & & & & & & & & & & & & & & & & & & & & & & \\
\hline & 0. & 0. & 0. & 0. & 0. & & 29 & & & 0 & 0 & & & & & & & & & & & & & & & & & & & & & & & & \\
\hline & 2. & 0.8 & 0. & $\begin{array}{l}0 . \\
0.1\end{array}$ & $l_{0}^{0}$ & & & & & & & & & & & & & & & & & & & & & & & & & & & & & & \\
\hline & & 0. & 0. & & & & & & & & & & & & & & & & & & & & & & & & & & & & & & & & \\
\hline & & & & & & & & & & & & & & & & & & & & & & & & & & & & & & & & & & & \\
\hline & & & & & & & 35 & & & & & & & & & & & & & & & & & & & & & & & & & & & & \\
\hline & & & & & & & & & & & & & & & & & & & & & & & & & & & & & & & & & & & \\
\hline & & & & 0. & & & & & & 0 & & & & & & & & & & & & & & & & & & & & & & & & & \\
\hline & 4. & 0. & 0. & 0 & 0 & & & 0 & & & & & & & & & & & & & & & & & & & & & & & & & & & 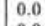 \\
\hline & 2.3 & 0. & 0.4 & 0. & 0. & & 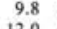 & 0 . & & 0. & & & & & & & & & & & & & & & & & & & & & & & & & \\
\hline & 3 & 0.2 & 0.2 & 0. & 0. & & 12.0 & 0. & & & & & & & & & & & & & & & & & & & & & & & & & & & 0.2 \\
\hline 2. & 1.3 & 0.0 & $\begin{array}{l}0.8 \\
0.8\end{array}$ & $\begin{array}{l}0.0 \\
0.0\end{array}$ & $\begin{array}{l}0.3 \\
0.0\end{array}$ & $\begin{array}{l}1.8 \\
1.2\end{array}$ & $\begin{array}{l}4.3 \\
7.2\end{array}$ & $\begin{array}{l}0.0 \\
0.0\end{array}$ & $\begin{array}{l}0.0 \\
0.0\end{array}$ & $\begin{array}{l}0.6 \\
0 .\end{array}$ & $\begin{array}{l}0.0 \\
0.0\end{array}$ & $\begin{array}{l}0.0 \\
0.0\end{array}$ & 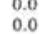 & & $\begin{array}{l}0 \\
1\end{array}$ & & & 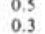 & $\begin{array}{l}0.2 \\
0.3\end{array}$ & & $\begin{array}{l}0.0 \\
0.0\end{array}$ & $\begin{array}{l}0.3 \\
0.7\end{array}$ & 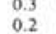 & 0 & & 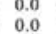 & & & & & $\begin{array}{l}0.0 \\
0.2\end{array}$ & & & & $\begin{array}{l}0.3 \\
0.0\end{array}$ \\
\hline 1. & 2.7 & 0.0 & 0 & 0.2 & 0.0 & 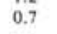 & 0.9 & 0.0 & 0.0 & 0.0 & 0.0 & 0.0 & 0.2 & 0.0 & 0.0 & 0.0 & 0.0 & 0.0 & 0.2 & 0 & 0.0 & 0.3 & 0.2 & 0.2 & & 0.0 & 0.0 & 0.9 & 2.7 & 0.0 & 0.2 & 0.7 & 0.0 & & 0.5 \\
\hline
\end{tabular}

Riedel (1978). Mineral grains on the slides were visually estimated to an order of magnitude. Results are plotted on a logarithmic scale in Figure 2.

At both Sites 607 and 609 there is a strong tendency for the radiolarians to be better preserved and more abundant where mineral grains are fewer, and vice versa. This evidence for dissolution of siliceous microfossils in the presence of high concentrations of terrigenous silt- and sand-size components has a parallel in the results reported by Johnson (1976), who found an inverse correlation between siliceous microfossil preservation and input of terrigenous silicates in sediments of the eastern tropical Pacific.

Exceptions to this general picture are more common at Site 607. In several of the samples from this site (e.g., 607-1-6, 40-42 cm, 607-2-5, 40-42 cm, 607-5-6, 40-42 $\mathrm{cm}, 607-7-6,40-42 \mathrm{~cm}$, and 607-10-3, 40-42 cm), low abundances of radiolarians, poorly preserved, are not associated with high concentrations of mineral grains.

\section{STATISTICAL ANALYSIS OF RADIOLARIAN ASSEMBLAGES}

Box-Jenkins time series analysis (Box and Jenkins, 1976) using the BMDP statistical software package (Dixon, 1983) was performed on the data to detect any trends in abundances of the counting groups occurring in the cores. This analysis confirms the impression given by the tabulation that the abundance of the Spongodiscus group decreases with time throughout the section at Site 609 . The reader can judge the degree of possible taxonomic heterogeneity of the forms grouped under this name by the fact that it includes the three species illustrated on Plate 15 of Nigrini and Moore (1979).

Cluster analysis was used to determine the similarity of samples and the tendency for the counted taxa to form groups based on the abundance data of the radiolarians at Sites 607 and 609 . The abundance data were standardized and analyzed using the average taxonomic 
Table 2. Abundance of Miocene radiolarians at Site 608.

\begin{tabular}{|c|c|c|c|c|c|c|c|c|c|c|c|c|c|c|c|c|c|c|}
\hline $\begin{array}{c}\text { Sample } \\
\text { (core-section, } \\
\text { interval in } \mathrm{cm} \text { ) }\end{array}$ & 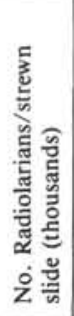 & 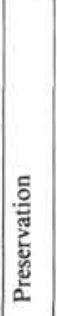 & 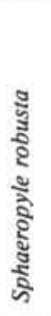 & 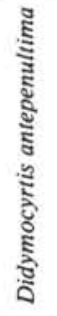 & 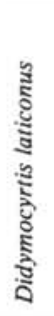 & 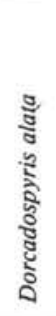 & 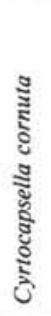 & 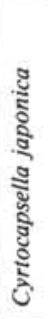 & 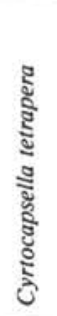 & 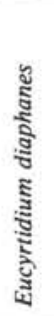 & 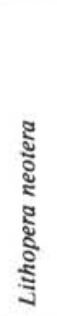 & 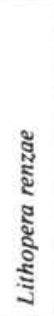 & 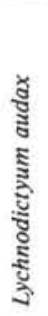 & 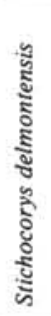 & 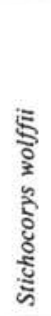 & 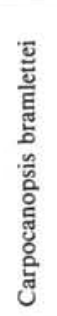 & 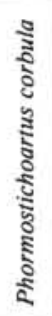 & 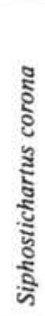 \\
\hline $29-2,40-42$ & 0.1 & P & $\mathrm{R}$ & $\mathbf{R}$ & $\mathbf{R}$ & $\mathrm{R}$ & $\mathrm{r}$ & . & $\mathbf{R}$ & - & $\mathrm{F}$ & . & $\mathbf{R}$ & F & $\mathbf{R}$ & - & . & . \\
\hline $29-6,40-42$ & 2.0 & G & $\mathbf{R}$ & + & $\mathbf{R}$ & - & + & - & $\mathrm{F}$ & r & $\mathrm{R}$ & - & $\mathrm{F}$ & F & . & - & - & . \\
\hline $29, \mathrm{CC}$ & 5.0 & P & - & + & $\mathbf{R}$ & - & $\mathbf{R}$ & - & $\mathrm{F}$ & $\mathrm{r}$ & $\mathrm{R}$ & . & $\mathbf{R}$ & F & - & - & . & $\mathbf{R}$ \\
\hline $30-2,40-42$ & 1.0 & M & - & - & $\mathbf{R}$ & - & - & - & $\mathrm{F}$ & $\mathrm{r}$ & - & - & - & $\mathbf{R}$ & . & - & - & $\mathrm{R}$ \\
\hline $30, \mathrm{CC}$ & 0.5 & M & - & - & $\mathbf{R}$ & - & - & - & - & - & $\mathbf{R}$ & - & + & F & . & - & $\mathrm{R}$ & . \\
\hline $31-2,40-42$ & 3.0 & G & - & - & $\mathbf{R}$ & - & - & - & $\mathrm{r}$ & $\mathbf{R}$ & $\mathrm{F}$ & $\mathrm{r}$ & $\mathrm{r}$ & F & - & . & . & $\mathbf{R}$ \\
\hline $31-6,40-42$ & 1.5 & G & - & - & $\mathrm{r}$ & - & $\mathbf{R}$ & - & $R$ & R & 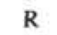 & + & - & F & - & + & . & R \\
\hline $31, \mathrm{CC}$ & 6.0 & G & - & - & r & . & $\mathrm{R}$ & $r$ & $\mathrm{~F}$ & $\vec{r}$ & $\mathrm{~F}$ & $\mathrm{r}$ & $\mathrm{R}$ & $\mathrm{F}$ & . & $\mathrm{r}$ & . & : \\
\hline $32-2,40-42$ & 1.0 & M & - & - & $r$ & - & $\mathrm{R}$ & - & $\mathrm{F}$ & $\mathrm{r}$ & $r$ & - & - & F & - & - & $\mathrm{T}$ & - \\
\hline $32-5,20-22$ & 0.1 & $\mathrm{P}$ & - & - & - & . & R & - & $\mathrm{F}$ & . & : & . & - & F & - & . & : & . \\
\hline
\end{tabular}

Note: Preservation: $\mathrm{G}=$ good, $\mathrm{M}=$ moderate, $\mathrm{P}=$ poor. Abundance: $-=$ looked for but not found; $+=\langle 0.01 \% ; \mathrm{r}=$ $0.01-0.1 \% ; \mathrm{R}=0.1 \%-1.0 \%$; and $\mathrm{F}=1.0 \%-10 \%$.

Table 3. Abundance of Miocene radiolarians at Site 610 .

\begin{tabular}{|c|c|c|c|c|c|c|c|c|c|c|c|c|c|c|c|c|c|c|c|c|c|c|c|}
\hline $\begin{array}{c}\text { Sample } \\
\text { (core-section, } \\
\text { interval in } \mathrm{cm} \text { ) }\end{array}$ & 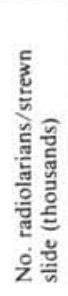 & 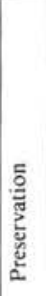 & 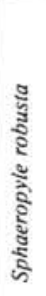 & 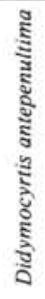 & 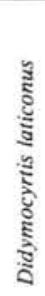 & 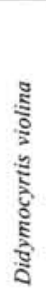 & 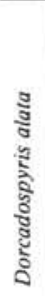 & 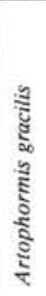 & 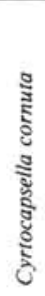 & 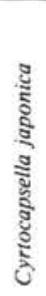 & 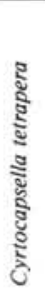 & 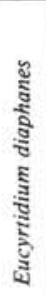 & 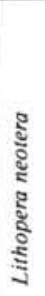 & 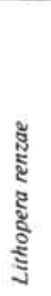 & 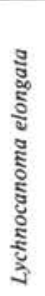 & $\begin{array}{l}\text { ปूँ } \\
\text { है } \\
\text { है } \\
\text { हूँ } \\
\text { हूँ } \\
\text { है }\end{array}$ & 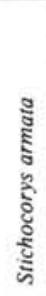 & 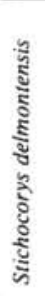 & 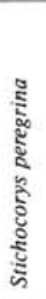 & 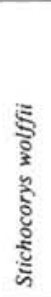 & 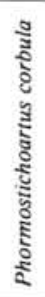 & 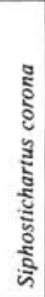 & Zone \\
\hline $15-2,46-48$ & 2.7 & G & $r$ & $\mathrm{r}$ & . & - & - & - & - & $r$ & . & - & $\mathrm{r}$ & . & . & - & - & $\mathrm{R}$ & $\mathrm{r}$ & R & $R$ & - & D. antepenultima \\
\hline $16-2,66-68$ & 3.0 & G & $\mathrm{r}$ & $\mathrm{r}$ & F & . & . & . & . & $\mathrm{F}$ & $\mathrm{R}$ & . & F & . & . & $\mathbf{R}$ & . & $\mathrm{R}$ & + & . & - & & \\
\hline $16-6,46-48$ & 3.0 & $\mathrm{G}$ & $r$ & $\therefore$ & $\mathrm{r}$ & . & . & - & . & $\mathrm{r}$ & $\ddot{R}$ & . & $\mathrm{r}$ & + & . & $\mathrm{R}$ & - & $\mathrm{r}$ & : & $\mathrm{r}$ & - & . & D. petterssoni/solidu \\
\hline $16, \mathrm{CC}$ & 2.5 & G & : & - & . & - & . & - & - & - & $\mathrm{F}$ & - & . & $\mathrm{t}$ & - & $\mathrm{r}$ & - & $\mathrm{r}$ & - & $\mathrm{r}$ & + & - & D. alata \\
\hline $17-3,46-48$ & 1.0 & $\mathrm{M}$ & $\mathrm{r}$ & - & - & . & $\mathrm{r}$ & - & $\mathrm{r}$ & - & $\mathrm{R}$ & - & . & $\mathrm{R}$ & . & : & . & $\mathrm{r}$ & . & $\mathrm{r}$ & . & . & \\
\hline $18-2,55-57$ & 0.8 & $\mathrm{P}$ & - & - & - & . & $\mathrm{R}$ & . & $\mathrm{R}$ & $\mathrm{R}$ & $\mathrm{F}$ & . & $\mathrm{R}$ & $\mathrm{R}$ & - & - & - & : & - & : & - & $\cdot$ & \\
\hline $19-3,40-42$ & 0.2 & $P$ & - & - & . & - & - & - & - & $\mathrm{F}$ & F & - & . & . & - & $\mathbf{R}$ & - & . & . & - & - & $\cdot$ & \\
\hline $20-2,106-108$ & 0.1 & P & - & - & - & - & - & - & $\mathbf{R}$ & $\mathrm{F}$ & $\mathbf{R}$ & - & . & - & - & $r$ & . & - & - & . & - & $\cdot$ & \\
\hline $21-2,46-48$ & 1.0 & $\mathrm{P}$ & - & . & . & + & . & $\mathrm{R}$ & $\mathrm{R}$ & $\mathrm{F}$ & $\mathrm{F}$ & - & . & . & $\mathbf{R}$ & $\mathrm{R}$ & . & . & . & . & . & . & \\
\hline $21, \mathrm{CC}$ & 0.5 & M & . & . & . & : & . & R & $\ddot{R}$ & $\mathrm{~F}$ & $\mathrm{~F}$ & . & . & . & R & : & . & - & . & - & - & - & \\
\hline $22, \mathrm{CC}$ & 0.2 & $\mathrm{P}$ & . & - & . & . & - & $\because$ & $\hat{R}$ & $\mathrm{R}$ & $\mathrm{R}$ & - & . & . & : & . & . & . & . & - & - & - & \\
\hline $23-2,46-48$ & 0.1 & $\mathrm{P}$ & - & - & - & - & . & - & R & $\mathrm{F}$ & $\ddot{R}$ & $\mathrm{R}$ & - & $\mathrm{R}$ ? & $\mathrm{R}$ & - & . & - & . & - & $\cdot$ & - & \\
\hline $23, \mathrm{CC}$ & 2.0 & M & . & . & . & $\mathrm{r}$ & - & $\mathbf{R}$ & $\ddot{R}$ & $\mathrm{~F}$ & $\hat{\mathrm{F}}$ & $\ddot{R}$ & . & 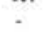 & & . & - & - & . & - & - & - & \\
\hline $24-3,46-48$ & 0.1 & $\mathrm{P}$ & - & - & . & : & - & $\because$ & $\because$ & $\mathrm{R}$ & $\mathrm{R}$ & $\ddot{R}$ & . & . & : & - & . & - & - & . & - & . & C. tetrapera \\
\hline $24, \mathrm{CC}$ & 0.3 & P & - & - & - & - & - & $\mathbf{R}$ & $\mathrm{R}$ & $\mathrm{F}$ & $\mathrm{F}$ & $\mathbf{R}$ & . & . & - & - & - & - & - & - & $\cdot$ & - & \\
\hline $25-3,36-38$ & 3.0 & G & $\mathrm{r}$ & . & . & $\mathrm{r}$ & . & $\hat{F}$ & $\hat{R}$ & . & $\mathrm{C}$ & $\mathrm{F}$ & . & . & & $\mathrm{r}$ & . & . & . & . & . & $\mathrm{R}$ & \\
\hline $25, \mathrm{CC}$ & 1.0 & M & : & & & : & - & . & R & $\mathbf{R}$ & $\mathrm{F}$ & $\mathrm{F}$ & . & . & : & R & - & . & - & - & - & $\mathrm{R}$ & \\
\hline $26-3,70-72$ & 0.8 & M & - & - & - & - & - & - & $\mathrm{F}$ & - & $\mathrm{F}$ & $\mathrm{F}$ & - & - & $\mathbf{R}$ & $\mathrm{R}$ & . & - & - & - & - & $\mathrm{R}$ & \\
\hline $26, \mathrm{CC}$ & 0.1 & $\mathrm{P}$ & - & - & - & . & - & - & : & - & $\mathrm{R}$ & : & . & . & . & . & . & - & & - & . & $\ddot{R}$ & \\
\hline $27-2,46-48$ & 2.0 & G & $\mathbf{R}$ & . & . & . & . & $\mathbf{R}$ & R & . & $\mathrm{C}$ & $\mathbf{R}$ & . & . & $\mathbf{R}$ & r & - & . & . & - & - & $\ddot{R}$ & \\
\hline $27-6,40-42$ & 0.1 & $P$ & - & . & - & - & . & - & $\mathbf{R}$ & - & $\mathrm{F}$ & $\mathrm{F}$ & - & . & - & . & $\mathrm{R}$ & - & - & - & - & R & \\
\hline $27, \mathrm{CC}$ & 1.5 & G & - & - & . & - & - & $\mathrm{r}$ & $\mathbf{R}$ & - & F & - & - & - & - & $\mathrm{r}$ & - & - & - & . & - & $\mathrm{R}$ & \\
\hline
\end{tabular}

distance coefficient (Sokal, 1961). For each analysis, a cophenetic correlation coefficient $\left(\mathrm{r}_{\mathrm{c}}\right.$; Sokal and Rohlf, 1962) was calculated to measure the amount of distortion of the original data by the dendrogram. The NTSYS package for multivariate statistics (Rohlf et al., 1984) was used.

Cluster analyses of the radiolarians produced similar results for both Sites 607 and 609 (Figs. 3 and 4, respec- tively). A tight cluster (A) containing most of the radiolarians reflects the low abundances of these radiolarians in the majority of the samples. A second small cluster (B) contains five groups (other Actinommids, Porodiscus group, Lophophaena group, Pseudodictyophimus group, and Botryostrobus group A) with intermediate abundances. Radiolarians with particularly high abundances (Spongodiscus group, Lithelius minor, Pylospira 


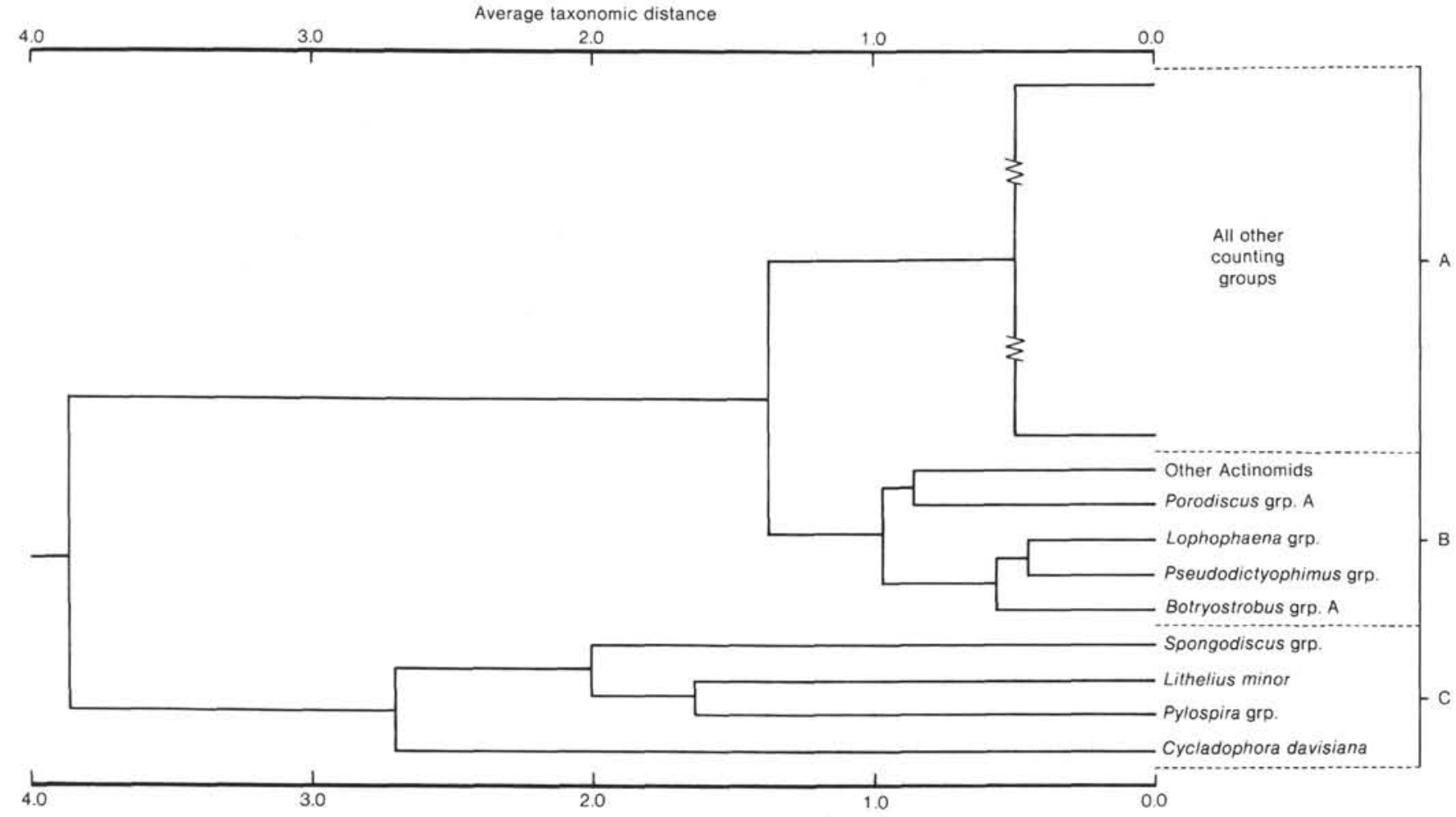

Figure 3. Dendrogram resulting from cluster analysis of radiolarian counting groups from Site $607\left(r_{c}=0.9814\right)$. See text for an explanation of A-C.

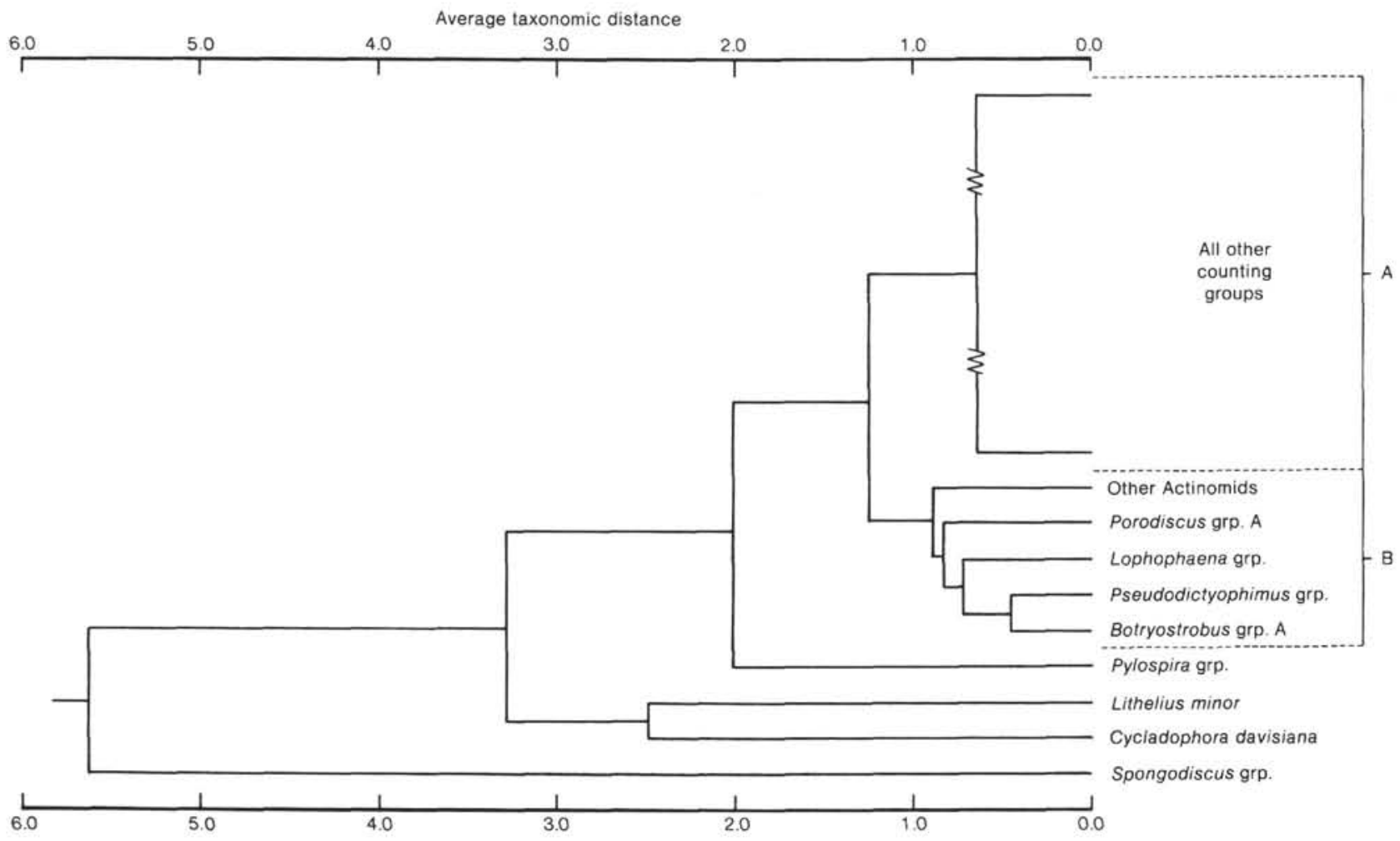

Figure 4. Dendrogram resulting from cluster analysis of radiolarian counting groups from Site $609\left(\mathrm{r}_{\mathrm{c}}=0.9910\right)$. See text for an explanation of $\mathrm{A}$ and $\mathrm{B}$. 
group, and Cycladophora davisiana) have very low similarity values compared to the other radiolarians, and form a third cluster (C) for Site 607.

Samples from Site 609 clustered into three distinct groups (Fig. 5). With the exception of Sample 609-13-5, 40-42 cm, all samples in clusters A and B were taken at the level of Section 609-12-2 and above. Additional samples would have to be examined to establish whether the apparent concentration of cluster A samples near the top of the column is meaningful. All samples in cluster C were taken at the level of Section 609-12-4 and below except for Sample 609-9-3, 40-42 cm. This indicates that the radiolarian assemblages are distinct in the parts of the section above and below Sample 609-12-2, 40-42 cm. From the tabulation it appears that the Stylodictya group is generally more abundant above this level than below it, whereas the Spongodiscus group was more abundant below this level. Theocalyptra bicornis was found in more than half the samples above this level and in none below it. We do not attempt to interpret these changes in paleoenvironmental terms because they are not very pronounced, and we have investigated only one other sequence (Site 607) with which Site 609 could be compared.

Cluster analysis of the samples from Site 607 did not produce any groups that can be readily interpreted. Samples are clustered into two distinct groups as shown in Figure 6, but these clusters show no stratigraphic coherence in the sequence, nor any clear relationship to radiolarian preservation.

These results indicate that analysis of much more closely spaced samples (so that several consecutive samples have similar assemblages) will be necessary for effective investigation of the paleoenvironmental signals in the

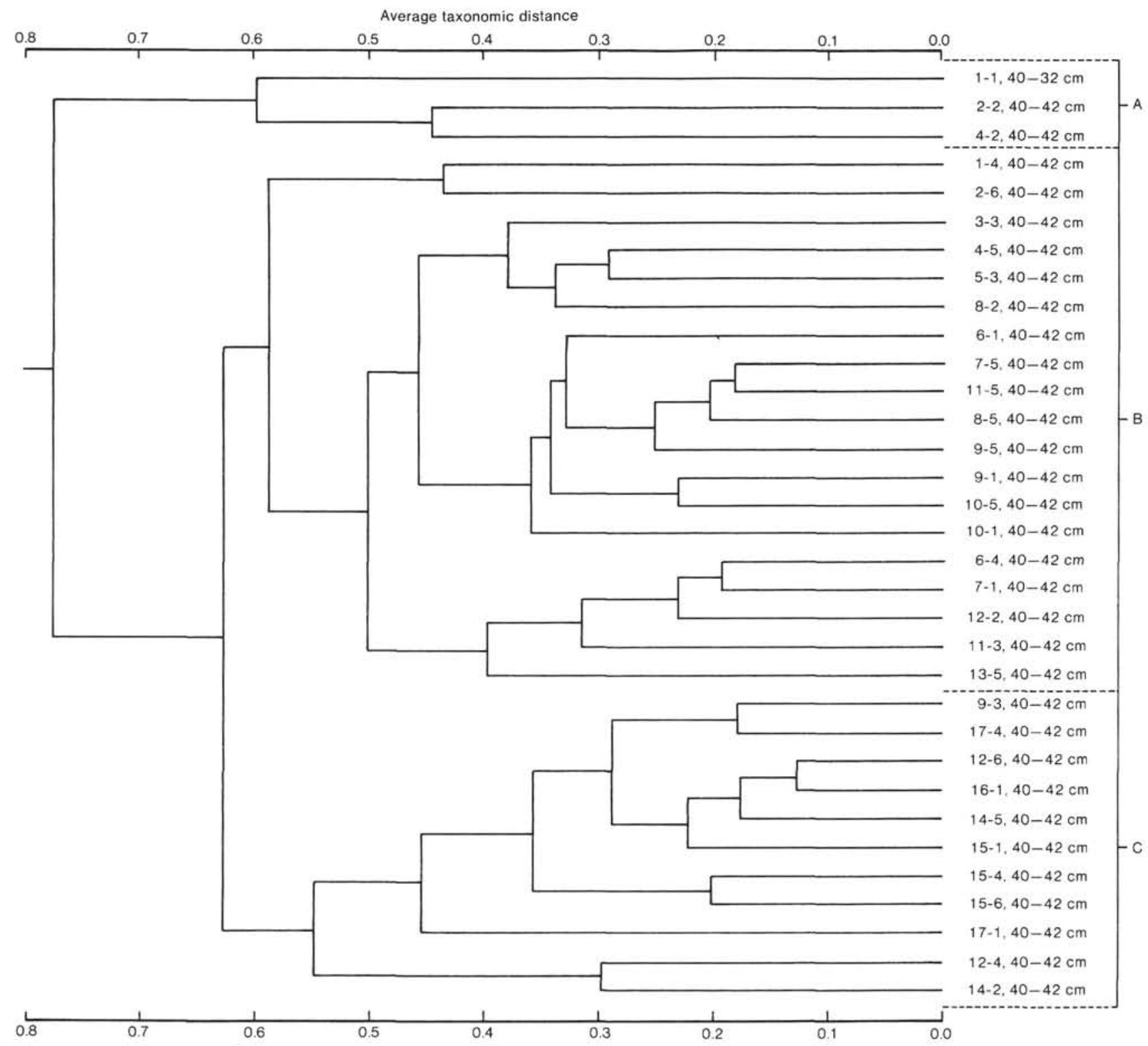

Figure 5. Dendrogram resulting from cluster analysis of samples from Site $609\left(r_{c}=0.7140\right)$. See text for an explanation of A-C. 


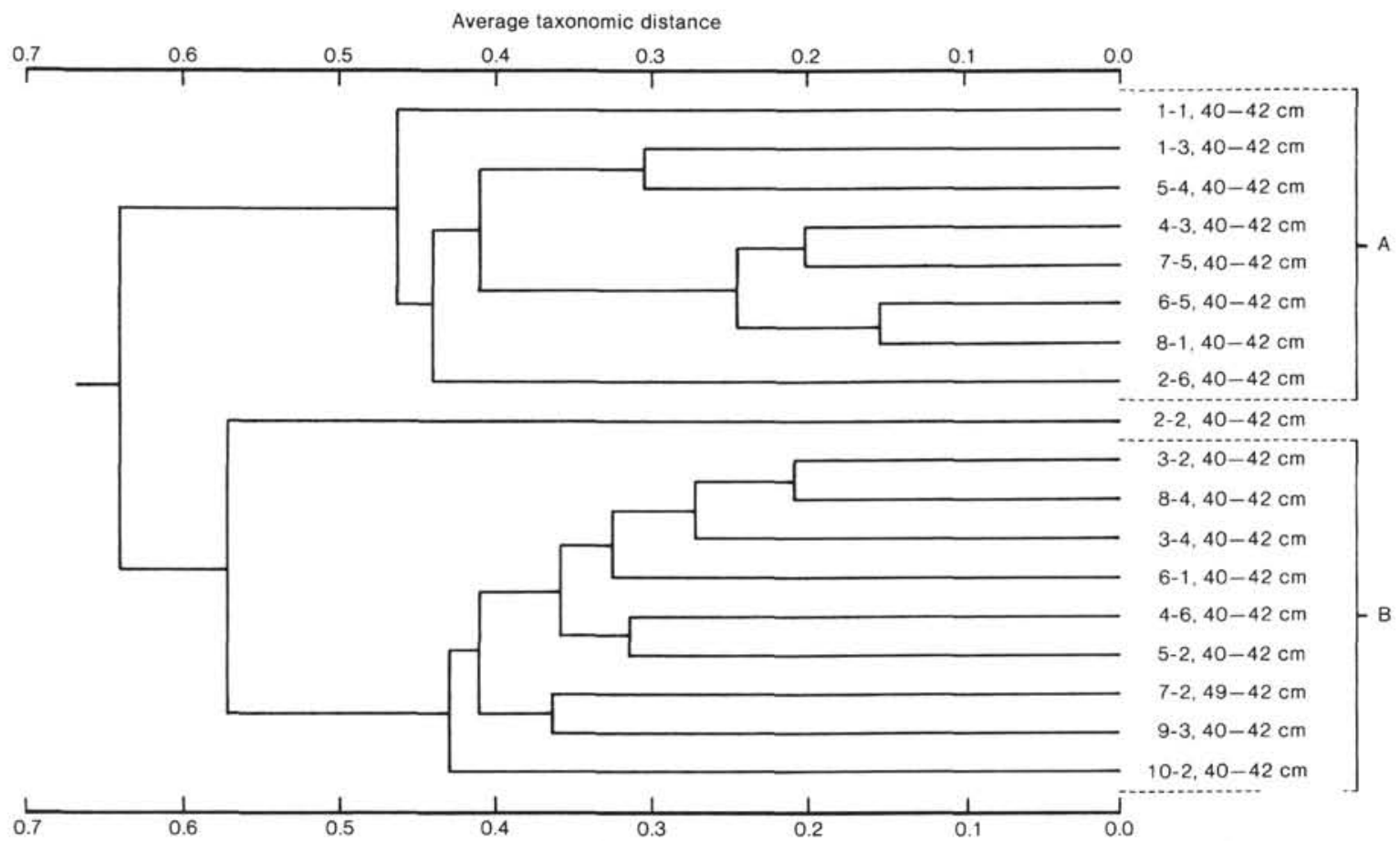

Figure 6. Dendrogram resulting from cluster analysis of samples from Site $607\left(r_{c}=0.8110\right)$. See text for an explanation of A and $\mathrm{B}$.

radiolarian record at Site 607 . Similarly, closer sampling at both Sites 607 and 609 would be required for a meaningful description and interpretation of radiolarian abundances and preservation, and would provide an improved basis for the statistical procedures.

\section{TAXONOMIC NOTES}

Listed below are the Miocene species used for stratigraphic interpretations, as well as the generally supraspecific groups used for counting the entire assemblages for paleoenvironmental interpretations.

\section{Family COLLOSPHAERIDAE Müller, 1858} Collosphaerid group

All collosphaerids are counted together in this study.

Family ACTINOMMIDAE Haeckel, 1862, emend, Sanfilippo and Riedel, 1980

Genus ACTINOMMA Haeckel, 1860a

Actinomma medianum Nigrini

Actinomma medianum Nigrini, 1967, p. 27, pl. 2, fig. 2a, b; Westberg-Smith and Riedel, 1985 , p. 485 , pl. 1, fig. 1a, b.

Genus ANOMALACANTHA Loeblich and Tappan, 1961 Anomalacantha dentata (Mast)

Heteracantha dentata Mast, 1910, p. 37, fig. 47

Anomalacantha dentata (Mast), Benson, 1966, p. 170, pl. 5, figs. 10, 11.

\section{Genus DRUPPATRACTUS Haeckel, 1887}

Druppatractus group A

Druppatractus group, Westberg-Smith and Riedel, 1985, p. 486, pl. 1, fig. 2.

Bipolar actinommid with pear-shaped medullary shell, and coarse pores on the cortical shell (less than eight across a half-equator). Polar spines may be conical or bladed, and equal or unequal in length; the lattice of the cortical shell is thick.

\section{Druppatractus group B}

Bipolar actinommid with pear-shaped medullary shell and ellipsoidal cortical shell. The lattice of the cortical shell is relatively thin, sometimes altogether missing, leaving only the unequal, bladed polar spines and the pear-shaped medullary shell. Members of this group resemble Druppatractus irregularis Popofsky (1912).

\section{Genus HEXACONTIUM Haeckel, 1881}

\section{Hexacontium group}

Hexacontium group A, Westberg-Smith and Riedel, 1985, p. 486, pl. I, fig. $4 a, b$.

Spherical form with two medullary shells and six to seven spines in three mutually perpendicular axes. The pores are small, closely spaced, more than eight across a half-equator. Some forms in this group tend to a cubic shape.

\section{Genus SPHAEROPYLE Dreyer, 1889} Sphaeropyle langii Dreyer

Sphaeropyle langii Dreyer, 1889, p. 13, pl. 4, fig. 54; Kling, 1973, p. 634, pl. 1, figs. 5-10, pl. 13, figs. 6-8; Westberg-Smith and Riedel, 1985 , p. 486 , pl. 1 , fig. 9.

\section{Sphaeropyle robusta Kling}

Sphaeropyle robusta Kling, 1973, p. 634, pl. 1, figs. 11, 12, pl. 6, figs. 9-13, pl. 13, figs. 1-5; Westberg-Smith and Riedel, 1985, p. 487, pl. 1 , fig. 8.

\section{Genus STYLATRACTUS Haeckel, 1887 Stylatractus universus Hays}

Stylatractus sp. Hays, 1965, p. 167, pl. 1, fig. 6 . Stylatractus universus Hays, 1970, p. 215.

Axoprunum angelinum (Campbell and Clark), Kling, 1973, p. 634, pl. 1 , figs. $13-16$, pl. 6 , figs. 14-18. 


\section{Genus STYLOSPHAERA Ehrenberg, 1847a}

Stylosphaera group

Stylosphaera spp., Westberg-Smith and Riedel, 1985, p. 487, pl. 2, fig. 1a, b.

Bipolar actinommid with spherical medullary shell and spherical to subspherical cortical shell with deep-set subcircular pores. Polar spines are cylindrical in cross section, and equal or unequal in length.

Other Actinommids

Any actinommid not previously categorized.

Family PHACODISCIDAE Haeckel, 1881

Phacodiscid group

Phacodiscid group, Westberg-Smith and Riedel, 1985, p. 487.

Family COCCODISCIDAE Haeckel, 1862, emend. Sanfilippo and Riedel, 1980

Subfamily ARTISCINAE Haeckel, 1881, emend, Riedel, 1967a

Artiscin group study.

All artiscins were counted together for the quantitative part of this

Genus DIDYMOCYRTIS Haeckel, 1860a

Didymocyrtis antepenultima (Riedel and Sanfilippo)

Ommatartus antepenultimus Riedel and Sanfilippo, 1970, p. 521, pl. 14, fig. 4; Westberg and Riedel, 1978, p. 22.

Didymocyrtis antepenultima (Riedel and Sanfilippo), Sanfilippo and Riedel, 1980, p. 1010; Sanfilippo et al., in press, fig. 8.6.

\section{Didymocyrtis laticonus (Riedel)}

Cannartus laticonus Riedel, 1959, p. 291, pl. 1, fig. 5; Westberg and Riedel, 1978, p. 20.

Didymocyrtis laticonus (Riedel), Sanfilippo and Riedel, 1980, p. 1010; Sanfilippo et al., in press, fig. $8.5 \mathrm{a}, \mathrm{b}$.

\section{Didymocyrtis violina (Haeckel)}

Cannartus violina Haeckel, 1887 , p. 358 , pl. 39 , fig. 10.

Didymocyrtis violina (Haeckel), Sanfilippo and Riedel, 1980, p. 1010; Sanfilippo et al., in press, fig. 8.3.

Family SPONGODISCIDAE Haeckel, 1862, emend. Riedel, 1967a Genus AMPHIRHOPALUM Haeckel, 1881

\section{Amphirhopalum group}

Amphirhopalum ypsilon Haeckel, 1887, p. 522; Nigrini, 1967, p. 35, pl. 3, fig. 3a-d (as Amphirrhopalum ypsilon).

Euchitonia virchowii Haeckel, 1862, p. 503-505, pl. 30, figs. 1-4.

Amphirhopalum virchowii (Haeckel), Dumitrică, 1973, p. 835, pl. 9, figs. 2 , 4 ; pl. 11 , fig. 6 ; pl. 21 , figs. $2-13$.

In the quantitative part of this report, both species of Amphirhopalum were counted together.

\section{Genus DICTYOCORYNE Ehrenberg, 1860}

\section{Dictyocoryne group}

Dictyocoryne group, Westberg-Smith and Riedel, 1985, p. 489, pl. 3, fig. 1 .

All three-armed spongodiscids except the Amphirhopalum group were counted together in this group. The specimens encountered here have simple, unchambered arms and no patagium.

\section{Genus PORODISCUS Haeckel, 1881}

\section{Porodiscus group}

Porodiscus group A, Westberg-Smith and Riedel, 1985, p. 489, pl. 2, fig. 4.

Porodiscus group B, Westberg-Smith and Riedel, 1985, p. 489, pl. 2, fig. 5.

A spongy disc with a variable number of regular or irregular concentric or spiral rings. The most common form has spongy material obscuring the concentric structure at the center of the disk, but specimens with distinct structure were also counted in this group.

Genus STYLODICTYA Ehrenberg, 1847a, emend. Kozlova in Petrushevskaya and Kozlova, 1972

\section{Stylodictya group}

Stylodictya spp., Westberg-Smith and Riedel, 1985, p. 489, pl. 2, fig. 6 .

A disk with concentric chambers, the innermost of which are distinctly scalloped.

Genus SPONGOCORE Haeckel, 1887

\section{Spongocore spp.}

Spongocore spp., Westberg-Smith and Riedel, 1985, p. 489, pl. 3, fig. 2. All cylindrical, bipolar spongodiscids.

\section{Genus SPONGODISCUS Ehrenberg, 1854}

\section{Spongodiscus group}

Spongodiscus group, Westberg-Smith and Riedel, 1985, p. 489, pl. 2, fig. $7 \mathrm{a}, \mathrm{b}$.

Any spongy discoidal form with no concentric structure.

Family PYLONIIDAE Haeckel, 1881

Genus HEXAPYLE Haeckel 1881

Hexapyle group

Pyloniids with six symmetrical gates.

Genus OCTOPYLE Haeckel, 1881

Octopyle group

Pyloniids with eight symmetrical gates.

Genus TETRAPYLE Müller, 1858

Tetrapyle group

Pyloniids with four symmetrical gates.

\section{Family LITHELIIDAE Haeckel, 1862 \\ Genus LARCOPYLE Dreyer, 1889}

Larcopyle group

Larcopyle group, Westberg-Smith and Riedel, 1985, p. 490, pl. 3, fig. $3 \mathrm{a}, \mathrm{b}$.

The internal spiral structure is enclosed in a smooth elliptical shell with regular outline and small pores. There may be a pylome visible at one end that may be surrounded by short spines.

\section{Genus LITHELIUS Haeckel, 1860b}

Lithelius minor Jörgensen

Lithelius minor Jörgensen, 1900, p. 65, pl. 5, fig. 24; Westberg-Smith and Riedel, 1985 , p. 490 , pl. 3 , fig. 5 .

\section{Genus PYLOSPIRA Haeckel, 1887}

Pylospira group

Pylospira group, Westberg-Smith and Riedel, 1985, p. 490, pl. 3, fig. $6 \mathrm{a}, \mathrm{b}$.

This broad counting group includes any litheliid with wide, loose whorls, and no enclosing shell. Tightly coiled litheliids were counted in the preceding group, L. minor.

Order NASSELLARIA Ehrenberg, 1875 Suborder SPYRIDA Ehrenberg, 1847b, emend. Petrushevskaya, 1971

\section{Genus CERATOSPYRIS Ehrenberg, 1847a}

\section{Ceratospyris group}

Ceratospyris group A, Westberg-Smith and Riedel, 1985, p. 491, pl. 3, fig. 9.

Ceratospyris group B, Westberg-Smith and Riedel, 1985, p. 491, pl. 3, fig. $8 \mathrm{a}, \mathrm{b}$. 
Ceratospyris group C, Westberg-Smith and Riedel, 1985, p. 491, pl. 3 , fig. 10.

Spyrids were very rare in this material, and this group contains any spyrid with a bilocular cephalic lattice.

\section{Genus DORCADOSPYRIS Haeckel, 1881 \\ Dorcadospyris alata (Riedel)}

Brachiospyris alata Riedel, 1959, p. 293, pl. 1, figs. 11, 12.

Dorcadospyris alata (Riedel), Sanfilippo et al., in press, fig. 10.7.

Genus THOLOSPYRIS Haeckel 1881, emend. Goll, 1969

Tholospyris rhombus (Haeckel)

Archicircus rhombus Haeckel, 1887, p. 942, pl. 81, fig. 7.

Tholospyris rhombus (Haeckel), Goll, 1972, p. 455, pl. 16, figs. 1-11.

\section{Genus ZYGOCIRCUS Bütschli, 1882}

Zygocircus group

Zygocircus group, Westberg-Smith and Riedel, 1985, p. 491, pl. 3, fig. 11.

Family PLAGONIIDAE Haeckel, 1881, emend. Riedel, 1967b Genus AMPHIPLECTA Haeckel, 1881

Amphiplecta group

Amphiplecta group, Westberg-Smith and Riedel, 1985, p. 491, pl. 4, fig. 1 .

Two-segmented plagoniid with a cylindrical, open cephalis and widely conical thorax.

\section{Genus ARACHNOCORALLIUM Haeckel, 1887}

\section{Arachnocorallium group}

Arachnocorallium group, Westberg-Smith and Riedel, 1985, p. 492, pl. 4, fig. 2.

One-segmented plagoniid consisting of an ovoid cephalis, the base of which is a narrow structure composed of the median bar and three protruding spines (dorsal and primary laterals).

\section{Genus CERATOCYRTIS Bütschli, 1882}

Ceratocyrtis group

Ceratocyrtis group, Westberg-Smith and Riedel, 1985, p. 492, pl. 4, fig. $3 a, b$.

Two-segmented plagoniid in which the small cephalis is separated from the hood-shaped thorax by a constriction.

\section{Genus LOPHOPHAENA Ehrenberg, 1847b}

Lophophaena group

Lophophaena group, Westberg-Smith and Riedel, 1985, p. 492, pl. 4, fig. $4 \mathrm{a}-\mathrm{e}$.

This large counting group (any two-segmented plagoniid in which the cephalis and thorax are nearly equal in volume) probably includes several genera. The cephalis is spherical to subspherical, and may or may not have horns. The thorax is open, conical to cylindrical, and may or may not have appendages. Pores are small, and irregular in size and shape.

\section{Genus PSEUDODICTYOPHIMUS Petrushevskaya, 1971}

\section{Pseudodictyophimus group}

Pseudodictyophimus group, Westberg-Smith and Riedel, 1985, p. 492 , pl. 4 , fig. 5 a, b.

Two-segmented form having three thoracic ribs that may extend beyond the margin of the thorax. The cephalis is small and may bear an apical horn. The second segment is usually open, conical to cylindrical, but it may be closed. The distinguishing characteristic in this group is the three thoracic ribs usually extending as feet.

\section{Sethophormin group $\mathbf{A}$}

Sethophormin group A, Westberg-Smith and Riedel, 1985, p. 492, pl. 4 , fig. 6.
Widely flattened conical skeleton, with three to four prominent ribs and a distinctly differentiated thoracic rim.

\section{Sethophormin group B}

Sethophormin group B, Westberg-Smith and Riedel, 1985, p. 492, pl.

4, fig. 7.

Widely flattened conical skeleton, with no prominent ribs nor distinctly differentiated thoracic rim.

\section{Other Plagoniids}

Other Plagoniids, Westberg-Smith and Riedel, 1985, p. 492, pl. 4, fig. $8 \mathrm{a}, \mathrm{b}$.

Any plagoniid that has not been categorized above.

Family THEOPERIDAE Haeckel 1881, emend. Riedel, 1967a Genus ARTOPHORMIS Haeckel, 1881

Artophormis gracilis Riedel

Artophormis gracilis Riedel, 1959, p. 300, pl. 2, figs. 12, 13.

\section{Genus BATHROPYRAMIS Haeckel, 1881}

Bathropyramis spp.

Slender, conical shell with heavy, quadrangular meshes.

Genus CORNUTELLA Ehrenberg, 1838

Cornutella spp.

Cornutella spp., Riedel et al., in press, pl. 4 , fig. 4.

Genus CYCLADOPHORA, Ehrenberg, 1847a

Cycladophora davisiana Ehrenberg

Cycladophora davisiana Ehrenberg, 1861, p. 297.

The subspecies described by Petrushevskaya (1967) were counted together in the quantitative part of this study.

\section{Genus CYRTOCAPSELLA Haeckel, 1887 Cyrtocapsella cornuta Haeckel}

Cyrtocapsa (Cyrtocapsella cornuta Haeckel, 1887, p. 1513, pl. 78, fig. 9.

Cyrtocapsella cornuta Haeckel, Sanfilippo and Riedel, 1970, p. 453, pl. 1, figs. 19-20.

\section{Cyrtocapsella japonica (Nakaseko)}

Eusyringium japonicum Nakaseko, 1963, p. 193, text-figs. 20-21, pl. 4 , figs. 1-3.

Cyrtocapsella japonica (Nakaseko), Sanfilippo and Riedel, 1970, p. 452 , pl. 1 , figs. $13-15$.

\section{Cyrtocapsella tetrapera Haeckel}

Cyrtocapsa (Cyrtocapsella) tetrapera Haeckel, 1887, p. 1512, pl. 78, fig. 5 .

Cyrtocapsella tetrapera Haeckel, Sanfilippo and Riedel, 1970, p. 453, pl. 1, figs. 16-18.

Genus CYRTOPERA Haeckel, 1881

Cyrtopera laguncula Haeckel

Cyrtopera laguncula Haeckel, 1887, p. 1451, pl. 75, fig. 10.

\section{Genus DICTYOPHIMUS Ehrenberg, 1847a}

\section{Dictyophimus group}

Dictyophimus crisiae Ehrenberg, 1854, p. 1476.

Dictyophimus hirundo (Haeckel) group, Nigrini and Moore, 1979, p. N35, pl. 22, figs. 2-4.

In the quantitative part of this study, both species of Dictyophimus were counted together. 
Genus EUCECRYPHALUS Haeckel, 1860b, emend.

Petrushevskaya, 1971

Eucecryphalus group

Eucecryphalus group, Westberg-Smith and Riedel, 1985, p. 492, pl. 4, fig. 10.

Three-segmented shell forming a wide cone. Circular pores are regularly spaced in transverse rows and increase in size distally. The abdomen varies from flatly expanded to conical. The most common member of this group is E. elizabethae (Haeckel) (Petrushevskaya, 1971, p. 224).

\section{Genus EUCYRTIDIUM Ehrenberg, 1847a, emend. Nigrini 1967}

\section{Eucyrtidium acuminatum (Ehrenberg)}

Eucyrtidium acuminatum Ehrenberg 1844, p. 84.

Eucyrtidium acuminatum (Ehrenberg), Nigrini, 1967, p. 81, pl. 8, fig.

3a, b.

Stichocyrtid with small conical abdomen, lumbar stricture not indented externally, four to five postabdominal segments of approximately equal length expanding distally.

\section{Eucyrtidium calvertense Martin}

Eucyrtidium calvertense Martin, 1904, p. 450-451, pl. 130, fig. 5.

Stichocyrtid of five of more segments, with distinct change in contour between second and third segments, and with longitudinally aligned pores set in deep furrows.

Eucyrtidium cienkowskii Haeckel group, Sanfilippo et al.

Eucyrtidium cienkowskii Haeckel, 1887, p. 1493, pl. 80, fig. 9.

Eucyrtidium cienkowskii group, Sanfilippo et al., 1973, p. 221, pl. 5 , figs. 7-11; Riedel et al., in press, pl. 4, fig. 6 .

Multisegmented stichocyrtid with small cephalis and thorax set off from the remaining segments by a distinct external constriction; the third segment is conical, longer than the remaining cylindrical segments. Small pores of nearly equal size are longitudinally aligned. Some specimens have winglike thoracic ribs.

\section{Eucyrtidium diaphanes Sanfilippo and Riedel}

Eucyrtidium diaphanes Sanfilippo and Riedel, in Sanfilippo et al., 1973 , p. 221 , pl. 5, figs. $12-14$.

\section{Other Eucyrtidium}

Any member of the genus that does not fall into one of the above groups.

Genus GONDWANARIA Petrushevskaya, 1974

Gondwanaria group A

Gondwanaria group A, Westberg-Smith and Riedel, 1985, p. 493, pl. 5 , fig. 4 .

\section{Gondwanaria group B}

Gondwanaria group B, Westberg-Smith and Riedel, 1985, p. 493, pl. 5 , fig. 5 .

\section{Genus LITHARACHNIUM Haeckel, 1860b}

\section{Litharachnium group}

Tent-shaped, or flattened cone with delicate quadrangular meshes.

Genus LITHOPERA Ehrenberg, 1847a

Lithopera neotera Sanfilippo and Riedel

Lithopera neotera Sanfilippo and Riedel, 1970, p. 454, pl. 1, figs. 2426, 28; Sanfilippo et al., in press, fig. 16.5a, b.

Lithopera renzae Sanfilippo and Riedel

Lithopera renzae Sanfilippo and Riedel, 1970, p. 454, pl. 1, figs. 21 23, 27; Sanfilippo et al., in press fig. 16.4a-c.
Genus LYCHNOCANOMA Haeckel, 1887

Lychnocanoma elongata (Vinassa de Regny)

Tetrahedrina elongata Vinassa de Regny, 1900, p. 243, pl. 2, fig. 31. Lychnocanoma elongata (Vinassa de Regny), Sanfilippo et al., 1973, p. 221, pl. 5, figs. 19-20; Sanfilippo et al., in press, fig. 19.1a, b.

Genus LYCHNODICTYUM Haeckel, 1881

Lychnodictyum audax Riedel

Lychnodictyum audax Riedel, 1953, p. 810, pl. 85, fig. 9; Sanfilippo et al., in press, fig. 21.2.

\section{Genus PTEROCANIUM Ehrenberg, 1847a}

Pterocanium group

Pterocanium group, Westberg-Smith and Riedel, 1985, p. 493, pl. 5, fig. 6 .

\section{Genus STICHOCORYS Haeckel, 1881 \\ Stichocorys armata (Haeckel)}

Cyrtophormis armata Haeckel, 1887, p. 1460, pl. 78, fig. 17.

Stichocorys armata (Haeckel), Riedel and Sanfilippo, 1971, p. 1595, pl. 2E, figs. 13-15.

\section{Stichocorys delmontensis (Campbell and Clark)}

Eucyrtidium delmontense Campbell and Clark, 1944, p. 56, pl. 7, figs. 19-20.

Stichocorys delmontensis (Campbell and Clark), Sanfilippo and Riedel, 1970, p. 451, pl. 1, fig. 9; Sanfilippo et al., in press, fig. 23.1a, b.

\section{Stichocorys peregrina (Riedel)}

Eucyrtidium elongatum peregrinum Riedel, 1953, p. 812, pl. 85, fig. 2. Stichocorys peregrina (Riedel), Riedel and Sanfilippo, 1970, p. 530.

\section{Stichocorys wolffii Haeckel}

Stichcorys wolffii Haeckel, 1887, p. 1479, pl. 80, fig. 10; Sanfilippo et al., in press, fig. 23.3a, b.

\section{Genus THEOCALYPTRA Haeckel, 1881}

Theocalyptra bicornis (Popofsky)

Pterocorys bicornis Popofsky, 1908, p. 228, pl. 34, figs. 7, 8.

Theocalyptra bicornis (Popofsky), Riedel, 1958, p. 240, pl. 4, fig. 4; Nigrini and Moore, 1979, p. N53, pl. 24, fig. 1.

Genus THEOCORYS Haeckel, 1881

\section{Theocorys group}

Theocorys group, Westberg-Smith and Riedel, 1985, p. 493, pl. 5, fig. 10 .

Three-segmented theoperid with open, although sometimes constricted, abdomen without a differentiated termination. Small, spherical cephalis bears a small apical horn. Pores are subcircular, of nearly equal size of both abdomen and thorax.

\section{Other Theoperids}

Any theoperid not accounted for in one of the preceding counting groups.

Family CARPOCANIIDAE Haeckel, emend. Riedel, 1967a Genus CARPOCANISTRUM Haeckel, 1887

Carpocanistrum group $\mathbf{A}$

Carpocanistrum group A, Westberg-Smith and Riedel, 1985, p. 493, pl. 5, fig. $11 \mathrm{a}, \mathrm{b}$.

Members of the genus with a smooth shell surface.

Carpocanistrum group B

Carpocanistrum group B, Westberg-Smith and Riedel, 1985, p. 494, pl. 5 , fig. 12. 
Form similar to Carpocanistrum group A in all respects except that the surface of the shell is rough.

\section{Genus CARPOCANOPSIS Riedel and Sanfilippo, 1971}

\section{Carpocanopsis bramlettei Riedel and Sanfilippo}

Carpocanopsis bramlettei Riedel and Sanfilippo, 1971, p. 1597, pl. $2 \mathrm{G}$, figs. $8-14$, pl. 8 , fig. 7 .

Family PTEROCORYTHIDAE Haeckel, emend. Riedel, 1967a, Moore, 1972

\section{Genus ANTHOCYRTIDIUM Haeckel, 1881}

Anthocyrtidium group

Anthocyrtidium group, Westberg-Smith and Riedel, 1985, p. 494, pl. 5 , fig. 15.

This group includes all two-segmented pterocorythids.

Genus LAMPROCYCLAS Haeckel, 1887

Lamprocyclas maritalis Haeckel

Lamprocyclas maritalis Haeckel, 1887, p. 1390, pl. 74, figs. 13, 14. Lamprocyclas maritalis maritalis Haeckel, Nigrini, 1967, p. 74, pl. 7 , fig. 5.

Lamprocyclas maritalis polypora, Haeckel, Nigrini, 1967, p. 76, pl. 7, fig. 6 .

Genus LAMPROCYRTIS Kling, 1973

Lamprocyrtis(?) hannai (Campbell and Clark)

Calocyclas hannai Campbell and Clark, 1944, p. 48, pl. 69, figs. 21, 22.

Lamprocyrtis(?) hannai (Campbell and Clark), Kling, 1973, p. 638, pl. 5, figs. $12-14$, pl. 12 , figs. 10-14.

\section{Genus PTEROCORYS Haeckel, 1881}

Pterocorys zancleus (Müller)

Eucyrtidium zanclaeum Müller, 1858, p. 41, pl. 6, figs. 1-3.

Pterocorys zancleus (Müller), Nigrini and Moore, 1979, p. N89, pl. 25 , fig. $11 \mathrm{a}, \mathrm{b}$.

\section{Genus THEOCORYTHIUM Haeckel, 1887}

Theocorythium trachelium group

Eucyrtidium trachelius Ehrenberg, 1872, p. 312.

Theocorys dianae Haeckel, 1887, p. 1416, pl. 69, fig. 11.

Theocorythium trachelium trachelium (Ehrenberg), Nigrini, 1967, p. 79 , pl. 8, fig. 2, pl. 9, fig. 2.

Theocorythium trachelium dianae (Haeckel), Nigrini, 1967, p. 77, pl. 8 , fig. 1a, b, pl. 9, fig. 1a, b.

Both subspecies were counted together in this study.

Family ARTOSTROBIIDAE Riedel, 1967b

Genus BOTRYOSTROBUS Haeckel 1887, emend. Nigrini, 1977

Botryostrobus aquilonaris (Bailey)

Eucyrtidium aquilonaris Bailey, 1856, p. 4, pl. 1, fig. 9.

Botryostrobus aquilonaris (Bailey), Nigrini, 1977, p. 246, pl. 1, fig. 1.

\section{Botryostrobus group A}

Botryostrobus group, Westberg-Smith and Riedel, 1985, p. 494, pl. 6, fig. $1 \mathrm{a}, \mathrm{b}$.

Shell with more than four segments, each with three or more transverse rows of small pores, and separated by deep constrictions. Cephalis bears an apical horn and cylindrical vertical tube. Outline of each segment is smooth and rounded.

\section{Genus PHORMOSTICHOARTUS Campbell, 1951, emend. Nigrini, 1977}

\section{Phormostichoartus corbula (Harting)}

Lithocampe corbula Harting, 1863, p. 12, pl. 1, fig. 21.

Phormostichoartus corbula (Harting), Nigrini, 1977, p. 252, pl. 1, fig. 10.

\section{Phormostichoartus group}

Phormostichoartus group, Westberg-Smith and Riedel, 1985, p. 494, pl. 6, fig. 2a, b.

Cylindrical shell with four segments, many rows of small, transversely aligned pores on third and fourth segments. There is no apical horn, and a well-developed, cylindrical vertical tube lies along the proximal thorax.

\section{Genus SIPHOCAMPE Haeckel, emend. Nigrini, 1977}

\section{Siphocampe group}

Siphocampe group, Westberg-Smith and Riedel, 1985, p. 494, pl. 6 , fig. 3 .

Three-segmented artostrobiid with a short vertical tube and no horn. Transverse rows of small pores are widely separated. Frequently, rounded constrictions separate the pore rows of the third segment.

Genus SIPHOSTICHARTUS Nigrini, 1977

Siphostichartus corona

Cyrtophormis (Acanthocyrtis) corona Haeckel, 1887, p. 1462, pl. 77, fig. 15.

Siphostichartus corona (Haeckel), Nigrini, 1977, p. 257, pl. 2, figs. 5-7.

Genus SPIROCYRTIS Haeckel, 1881, emend. Nigrini, 1977

Spirocyrtis group

Spirocyrtis group, Westberg-Smith and Riedel, 1985, p. 494, pl. 6, fig. 4 .

Multisegmented artostrobiid with prominent flared (duck-billed) vertical tube and apical horn. Segments with angular constrictions expand distally, presenting a stepped profile.

Family CANNOBOTRYIDAE Haeckel, emend. Riedel, 1967a

Cannobotryid group

All members of the family were counted together.

Incertae sedis

Genus CARPOCANARIUM Haeckel, 1887

\section{Carpocanarium group}

Carpocanarium group, Westberg-Smith and Riedel, 1985, p. 494, pl. 6, fig. 5 .

Two-segmented form with hemispherical cephalis separated from an ovate thorax by a well-defined stricture. Sometimes a small lateral cephalic tube is observed, and in some specimens there are three small, winglike thoracic ribs. Pores are widely spaced, of nearly equal size.

\section{REFERENCES}

Bailey, J. W., 1856. Notice of microscopic forms found in the soundings of the Sea of Kamtschatka-with a plate. Am. J. Sci. Arts, Ser. 2, $22(64$ [Vol. 72]), pp. 1-6, pl. 1.

Benson, R. N., 1966. Recent radiolaria from the Gulf of California [Ph.D. dissert.] Minnesota University, Minneapolis, Minnesota.

Box, G. E. P., and Jenkins, G. M., 1976. Time Series Analysis, Forecasting and Control: San Francisco (Holden Day).

Bütschli, O., 1882. Beiträge zur Kenntnis der Radiolarienskelette, insbesondere der der Cyrtida. Z. Wissen. Zool., 36:485-540.

Campbell, A. S., 1951. New genera and subgenera of Radiolaria. $J$. Paleontol., 25(4):527-530.

Campbell, A. S., and Clark, B. L., 1944. Miocene radiolarian faunas from southern California. Geol. Soc. Am. Spec. Pap., 51:1-76.

Dixon, W. J., 1983. Biomedical Statistical Software (BMDP): Los Angeles (University of California Press).

Dreyer, F., 1889. Die Pylombildungen in vergleichendanatomischer und entwicklungs-geschichtlicher Beziehung bei Radiolarien und bei Protisten überhaupt. Jenaische Z. Naturwiss., 23:1-138.

Dumitricǎ, P., 1973. Cretaceous and Quaternary Radiolaria in deep sea sediments from the northeast Atlantic Ocean and Mediterranean Sea. In Ryan, W. B. F., Hsü, K. J., et al., Init. Repts. DSDP, 13, Pt. 2: Washington (U.S. Govt. Printing Office), 829-901.

Ehrenberg, C. G., 1838. Über die Bildung der Kreidefelsen und des Kreidemergels durch unsichtbare Organismen. K. Akad. Wiss. Berlin, Abh., pp. 59-147, pls. 1-4. 
1844. Über 2 neue Lager von Gebirgsmassen aus Infusorien als Meeres-Absatz in Nord-Amerika und eine Vergleichung derselben mit den organischen Kreide-Gebilden in Europa und Afrika. K. Preuss, Akad. Wiss. Berlin, Ber., pp. 57-97.

1847a. Über eine halibiolithische, von Herrn R. Schomburgk entdeckte, vorherrschend aus mikroskopischen Polycystinen gebildete, Gebirgsmasse von Barbados. K. Preuss. Akad. Wiss. Berlin, Ber., pp. 382-385.

1847b. Über die mikroskopischen kieselschaligen Polycystinen als mächtige Gebirgsmasse von Barbados und über das Verhältniss der aus mehr als $\mathbf{3 0 0}$ neuen Arten bestehenden ganz eigenthümlichen Formengruppe jener Felsmasse zu den jetzt lebenden Thieren und zur Kreidebildung. Eine neue Anregung zur Erforschung des Erdlebens. K. Preuss. Akad. Wiss. Berlin, Ber., pp. 4060 .

1854. Die systematische Charakteristik der neuen mikroskopischen Organismen des tiefen atlantischen Oceans. $K$. Preuss. Akad. Wiss. Berlin, Ber., pp 236-250.

1860. Über den Tiefgrund des stillen Oceans zwischen Californien und den Sandwich-Inseln aus bis 15600' Tiefe nach Lieutenant Brooke. K. Preuss. Akad. Wiss. Berlin, Monatsber, pp, 819-833.

1861. Über die Tiefgrund-Verhältnisse des Oceans am Eingange der Davisstrasse und bei Island. K. Preuss. Akad. Wiss. Berlin, Monatsber., pp. 275-315.

1872. Mikrogeologische Studien als Zusammenfassung der Beobachtungen des kleinsten Lebens der Meeres-Tiefgründe aller Zonen und dessen geologischen Einfluss. K. Preuss. Akad. Wiss. Berlin, Monatsber., pp. 265-322.

1875. Fortsetzung der mikrogeologischen Studien als Gesammt-Uebersicht der mikroskopischen Paläontologie gleichartig analysirter Gebirgsarten der Erde, mit specieller Rücksicht auf den Polycystinen-Mergel von Barbados. K. Preuss. Akad. Wiss. Berlin, Abh., pp. 1-225.

Goll, R. M., 1969. Classification and phylogeny of Cenozoic Trissocyclidae (Radiolaria) in the Pacific and Caribbean basins. Part II. J. Paleontol., 43(2):322-339.

1972. Systematics of eight Tholospyris taxa (Trissocyclidae, Radiolaria). Micropaleontology, 18(4):443-475.

Haeckel, E., 1860a. Über neue, lebende Radiolarien des Mittelmeeres und... die dazu gehörigen Abbildungen. K. Preuss. Akad. Wiss. Berlin, Monatsber., pp. 794-817.

1860b. Fernere Abbildungen und Diagnosen neuer Gattungen und Arten von lebenden Radiolarien des Mittelmeeres. K. Preuss. Akad. Wiss. Berlin, Monatsber., pp. 835-845.

, 1862. Die Radiolarien (Rhizopoda Radiolaria): Berlin (Reimer).

1881. Entwurf eines Radiolarien-Systems auf Grund von Studien der Challenger-Radiolarien. J. Z. Naturwiss, 15:418-472. 1887. Report on the Radiolaria collected by H.M.S. Challenger during the years 1873-1876. In Report on the Scientific Results of the Voyage of the H.M.S. Challenger, Zoology, 18.

Harting, P., 1863. Bijdrage tot de kennis der mikroskopische Fauna en Flora van de Banda-Zee. Verh. K. Acad. Wetensch., Amsterdam, pp. 2-54.

Hays, J. D., 1965. Radiolaria and late Tertiary and Quaternary history of Antarctic Seas. In Biology of Antarctic Seas, II: Am. Geophys. Union, Antarctic Res. Ser., 5:125-184.

1970. Stratigraphy and evolutionary trends of Radiolaria in north Pacific deep sea sediments. In Hays, J. D., (Ed.), Geological Investigations of the North Pacific, Geol. Soc. Am. Mem., 126: 185-218.

Johnson, D. A., 1976. Cenozoic radiolarians from the central Pacific, DSDP Leg 33. In Schlanger, S. O., Jackson, E. D., et al., Init. Repts. DSDP, 33: Washington (U.S. Govt. Printing Office), 425437.

Jörgensen, E., 1900. Protophyten und Protozöen im Plankton aus der norwegischen Westküste. Bergens Mus. Aarbog (1899), pp. 1-112.

Kling, S. A., 1973. Radiolaria from the eastern North Pacific, Deep Sea Drilling Project Leg 18. In Kulm, L. D., von Huene, R., et al., Init. Repts. DSDP, 18: Washington (U.S. Govt. Printing Office), pp. 617-671.

Loeblich, A. R., and Tappan, H., 1961. Remarks on the systematics of the Sarkodina (Protozoa), renamed homonyms and new validated genera. Proc. Biol. Soc. Washington, 74:213-234.
Martin, G. C., 1904. Radiolaria: Baltimore (Maryland Geological Survey, The John Hopkins Press), pp. 447-459.

Mast, H., 1910. Die Astrophaeriden der Deutschen Tiefsee-Expedition. Inaugural-Dissertation zur Erlangung der Doktorwurde, Universität Tübingen, pp. 1-68, pl. 1-8.

Moore, T. C., Jr., 1972. Mid-Tertiary evolution of the radiolarian genus Calocycletta. Micropaleontology, 18:144-152.

Müller, J., 1858. Über die Thalassicollen, Polycystinen und Acanthometren des Mittelmeeres. K. Preuss. Akad. Wiss. Berlin, Abh., pp. 1-62.

Nakaseko, K., 1963. Neogene Cyrtoidea (Radiolaria) from the Isozaki Formation in Ibaraki Prefecture, Japan. Sci. Rep. College of General Education, Osaka University, 12(2):165-198.

Nigrini, C. A., 1967. Radiolaria in pelagic sediments from the Indian and Atlantic Oceans. Scripps Inst. Oceanog. Bull. 11:1-125.

1977. Tropical Cenozoic Artostrobiidae (Radiolaria). Micropaleontology, 23(3):241-269.

Nigrini, C., and Moore, T. C., 1979. A guide to modern Radiolaria. Cushman Found. Foraminif. Res. Spec. Publ., 16:(S1-S142, N1N106), i-xii.

Petrushevskaya, M. G., 1967. Radiolarians of the orders Spumellaria and Nasellaria of the Antarctic region. (in Russian) Rezultaty biologicheskikh issledovanii Sovetskoi antarkticheskoi ekspeditsii (19551958). Sssledovaniya fauny mozei IV (XII), Vol. 3, pp. 5-183.

1971. On the natural system of polycystine Radiolaria (Class Sarcodina). In Farinacci, A. (Ed.), Proc. 2nd Plank. Conf., Rome (Tecnoscienza), pp. 981-982.

1974. Radiolarians of the Antarctic Waters and Their Distribution in the Sediments of Shelf: Nauka (Academy of Sciences, Institute of Geology, Leningrad, USSR), pp. 40, 41.

Petrushevskaya, M. G., and Kozlova, G. E., 1972. Radiolaria: Leg 14, Deep Sea Drilling Project. In Hayes, D. E., Pimm, A. C., et al., Init. Repts. DSDP, 14: Washington (U.S. Govt. Printing Office). 495-648.

Popofsky, A., 1908. Die Radiolarien der Antarktis (mit Ausnahme der Tripyleen). Deutsche Südpolar-Exped. 1901-1903, 10:183-305.

1912. Die Sphaerellarien des Warmwassergebietes. Deutsch Südpolar-Exped., 1901-1903, 13:73-159.

Riedel, W. R., 1953. Mesozoic and late Tertiary radiolaria of Rotti. $J$. Paleontol., 27(6):805-813.

1958. Radiolaria in Antarctic sediments. Repts, B.A.N.Z. Antarctic Research Expedition, Ser. B, 6(10):217-255.

1959. Oligocene and Lower Miocene Radiolaria in tropical Pacific sediments. Micropaleontology, 5(3):285-302. 1967a. Subclass Radiolaria. In Harland, W. B., Holland, C. H., House, M. R. et al. (Eds.), The Fossil Record: London (Geological Society of London), pp. 291-298.

1967b. Some new families of Radiolaria. Proc. Geol. Soc. London, 1640:148-149.

Riedel, W. R., and Sanfilippo, A., 1970. Radiolaria, Leg 4, Deep Sea Drilling Project. In Bader, R. G., Gerard, R. D., et al., Init. Repts. DSDP, 4: Washington (U.S. Govt. Printing Office), 503-575.

1971. Cenozoic Radiolaria from the western tropical Pacific, Leg 7. In Winterer, E. L., Riedel, W. R., et al., Init. Repts. DSDP, 7, Pt. 2: Washington (U.S. Govt. Printing Office), 15291672 .

1978. Radiolaria. In Micropaleontological counting methods and techniques-an exercise on an eight-meter section of the lower Pliocene of Capo Rosello, Sicily. Utrecht Micropaleontol. Bull., 17:81-128.

Riedel, W. R., Westberg-Smith, M. J., and Budai, A., in press. Late Neogene Mediterranean radiolaria from the point of view of paleoenvironment. In Stanley, D. J., and Wetzel, F. (Eds.), Geological Evolution of the Mediterranean Basin: New York (Plenum Press),

Rohlf, F. J., Kishpaugh, J., and Kirk, D., 1984. Numerical Taxonomy System of Multivariate Statistical Programs (NT-SYS): Stoney Brook (State University of New York at Stoney Brook).

Sanfilippo, A., Burckle, L. H., Martini, E., and Riedel, W. R., 1973. Radiolarians, diatoms, silicoflagellates, and calcareous nannofossils in the Mediterranean Neogene. Micropaleontology, 19(2):209234.

Sanfilippo, A., and Riedel, W. R., 1970. Post-Eocene "closed" theoperid radiolarians. Micropaleontology, 16(4):446-462. 1980. A revised generic and suprageneric classification of the Artiscins (Radiolaria). J. Paleontol., 54(5):1008-1011. 
Sanfilippo, A., Westberg-Smith, J., and Riedel, W. R., in press. Cenozoic Radiolaria. In Bolli, H. M., Perch-Nielsen, K., and Saunders, J. B., (Eds.) Plankton Stratigraphy: Cambridge (Cambridge University Press).

Sokal, R. R., 1961. Distance as a measure of taxonomic similarity. Systematic Zoology, 10:70-79.

Sokal, R. R., and Rohlf, F. J., 1962. The comparison of dendrograms by objective methods. Taxon, 11:33-40.

Vinassa de Regny, P. E., 1900. Radiolari miocenici italiani. Mem. $R$. Accad. Sci. Ist. Bologna, Ser. 5, 8:227-257 (565-595), pls. 1-3.
Westberg, M. J., and Riedel, W. R., 1978. Accuracy of radiolarian correlations in the Pacific Miocene. Micropaleontology, 24(1):123.

Westberg-Smith, M. J., and Riedel, W. R., 1985. Radiolarians from the western margin of the Rockall Plateau: Deep Sea Drilling Project Leg 81. In Roberts, D. G. and Schnitker, D., et al., Init. Repts. DSDP, 81: Washington (U.S. Govt. Printing Office), 479-501.

Date of Initial Receipt: 1 February 1985

Date of Acceptance: 25 June 1985 